\title{
One-dimensional inverse scattering problem for optical coherence tomography
}

\author{
O Bruno and J Chaubell \\ California Institute of Technology, 1200 E California Blvd, Pasadena, CA 91125, USA \\ E-mail: bruno@acm.caltech.edu
}

Received 13 February 2004, in final form 4 January 2005

Published 23 February 2005

Online at stacks.iop.org/IP/21/499

\begin{abstract}
Optical coherence tomography is a non-invasive imaging technique based on the use of light sources exhibiting a low degree of coherence. Low-coherence interferometric microscopes have been successful in producing internal images of thin pieces of biological tissue; typically samples of the order of $1 \mathrm{~mm}$ in depth have been imaged, with a resolution of the order of $10 \mu \mathrm{m}$ in some portions of the sample. In this paper we deal with the imaging problem of determining the internal structure of a multi-layered sample from backscattered laser light and low-coherence interferometry. In detail, we formulate and solve an inverse problem which, using the interference fringes that result as the back scattering of low-coherence light is made to interfere with a reference beam, produces maps detailing the values of the refractive index within the imaged sample. Unlike previous approaches to the OCT imaging problem, the method we introduce does not require processing at data collection time, and it produces quantitatively accurate values of the refractive indexes within the sample from back-scattering interference fringes only.
\end{abstract}

\section{Introduction}

The problem of imaging material bodies by means of waves and rays has had a tremendous impact on a wide variety of fields; here we are concerned with a relatively new imaging technique, optical coherence tomography (OCT) [1], which has thus far been used for imaging in biological and medical applications. This technique, which is based in interferometry, takes advantage of the low-coherence properties of diode-laser light sources to image selectively (and sequentially) prescribed points within a volumetric sample. Low-coherence interferometric microscopes [2, 3] have been successful in producing internal images of thin pieces of biological tissue; typically samples of the order of $1 \mathrm{~mm}$ in depth have been imaged, with a resolution of the order of $10 \mu \mathrm{m}$ in some portions of the sample. Such images have typically 
been produced through direct renderings of raw data: the intensities of certain interference fringes as functions of the position of the light focus within the sample; quite generally, limited post-processing of these data has been used.

In this paper we formulate and solve, in a mathematically rigorous manner, an inverse problem based on Maxwell's equations which, using OCT data (i.e., interference fringes that result as the back-scattering of low-coherence light is made to interfere with a reference beam), produces maps detailing the values of the refractive index within the imaged sample. As we will show, the coherence properties of light, which certainly play a central role in previous OCT imaging strategies (such as those proposed in $[14,15]$ ), can also be exploited to solve efficiently the fringes-to-indexes inverse problem under consideration. In particular we show that, using wide light-frequency bands, a certain OCT inverse problem we introduce allows for accurate rendering of refractive index distributions for layered structures. Once obtained, such a map of the refractive index variations may be useful in a variety of ways [2]; in particular, a straightforward display of this map yields an image of the internal structure of the sample. The advantages of an approach based on the Maxwell equations are manifold. Notably, such full-wave treatments allow for the consideration of various loss mechanisms such as scattering and absorption, and thus result in images that remain faithful throughout the body of the sample. Our present discussion, which accounts fully for the statistical nature of the coherence phenomenon, is restricted to one-dimensional parallel-layer samples; the methods and results of this paper have been announced in [4].

OCT devices have been used previously for evaluation of refractive index maps in layered samples containing a small number of layers [2, 5-7]. (Of course, OCT depiction of samples containing large numbers of pixels have been produced before, but those only show, for a given pixel, the interference brightness level and not the value of the refractive index corresponding to that pixel.) Previous OCT approaches for evaluation of refractive indexes, which are based on approximate geometrical considerations and basic laws of geometrical optics, sequentially yield the thickness and refractive index of each layer within a layered structure from two measured quantities. In [5, 6], for example, the measured information used for thickness/index evaluation is the sample displacement required to obtain a maximum reflected intensity and the mirror displacement giving a maximum for the interference fringe intensities. Clearly, such methods are rather slow, since, for determination of the refractive index value giving rise to a given peak, they need an accurate determination and processing of such maxima at measurement time; accordingly, only structures consisting of the order $\approx 10$ layers or less have heretofore been dealt with by means of such methods. The technique introduced in this paper accounts rigorously for all physical effects and, in particular, it does not rely on approximations such as those mentioned above. More importantly, the present solver produces solutions for inverse problems from back-scattering interference fringes only and, unlike those of [5-7] it does not require processing at data collection time. Thus, the algorithm described in this text can produce solutions for inverse problems including thousands of layers, and it results in quantitatively accurate renderings of the refractive index maps within the sample.

The importance of parallel-layer geometries is manifold: not only do they arise in a range of important engineering applications [5] (e.g., for quality control of films of various materials), but they also provide useful testbeds for techniques which, like those presented here, should extend to more general configurations. We note that, when layers are not planar our techniques do not apply directly, however-since, as it impinges on a curved interface, light is backscattered in a wide range of directions, which depend on the unknown geometry. We expect that appropriate representations of surface layers and new approaches to collection of scattered light will enable a generalization of the present techniques for evaluation of interface normals, and thus for volumetric imaging of fully two- and three-dimensional bodies. 


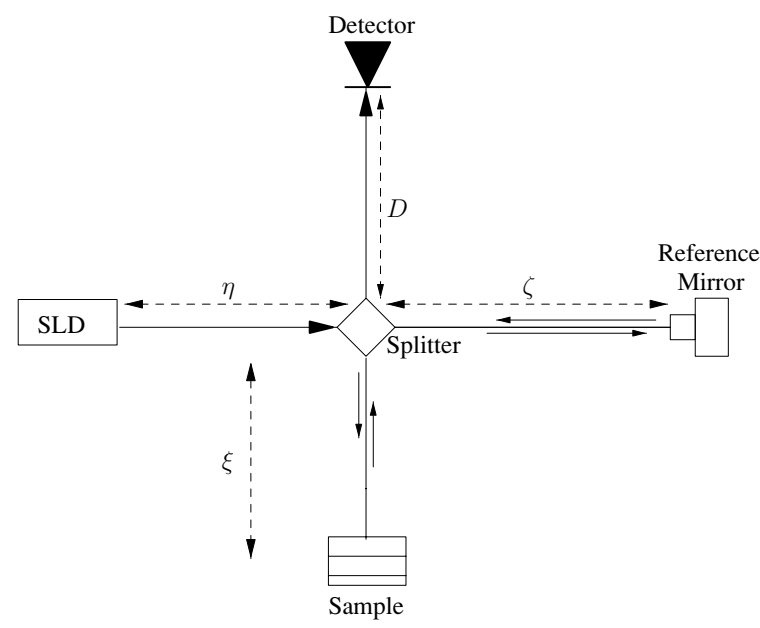

Figure 1. Optical coherence microscope.

The remainder of this paper is organized as follows. In section 2 we focus on the OCT model and present our strategy for solution of the associated direct problem. We show that, for a multi-layer structure, a rigorous geometrical optics method which takes advantage of the coherence properties of OCT light sources can be used to produce a fast direct-problem solver-as required by our inverse-problem algorithm. In section 3 we then introduce our inverse solver and in section 4 , finally, present a variety of numerical results.

\section{Mathematical formulation}

In this section we present the governing principles of the OCT technique (for a typical OCT device $[3,16]$, see figure 1 ), we describe our inverse problem, and formulate the fast algorithm we use for the solution of the associated direct problem.

\subsection{Layered media}

In this paper we assume that the polarization of the incident beam is transverse electric (TE), that is, that the electric field is orthogonal to the plane $(x, z)$, in which variations of the complex refractive index $n(z)+\mathrm{i} \kappa(z)$ occur. The wave equation describing the propagation of linearly polarized TE light in our dielectric medium is given by

$$
\left[\frac{\partial^{2}}{\partial z^{2}}+\frac{\partial^{2}}{\partial x^{2}}+k_{0}^{2}(n(z)+\mathrm{i} \kappa(z))^{2}\right] u(x, z)=0 .
$$

Here $k_{0}=2 \pi / \lambda=\omega / c$ is the wavenumber, $\lambda$ and $c$ denote the wavelength and speed of light in vacuum, respectively, $n(z)+\mathrm{i} \kappa(z)=\sqrt{\mu \epsilon(z)}$ is the refractive index of the medium-with $\mu$ the magnetic permeability (a constant throughout space for the non-magnetic samples under consideration) and $\epsilon=\epsilon(z)$ the dielectric constant of the medium varying in the $z$ directionand where $u(x, z)=E_{y}(x, z)$ is the $y$-component of the electric field [17]. Throughout this paper we assume the coefficients $\epsilon(z)$ and $n(z)+i \kappa(z)$ are locally constant, and, thus, they define a layered structure consisting of a finite set of parallel planar layers. Equation (1) is to be understood in the weak sense, so that continuity of $u$ and $\frac{\mathrm{d} u}{\mathrm{~d} z}$ across discontinuity surfaces is always assumed. 


Region $1 \quad$ Region $2 \quad$ Region 3

(3)

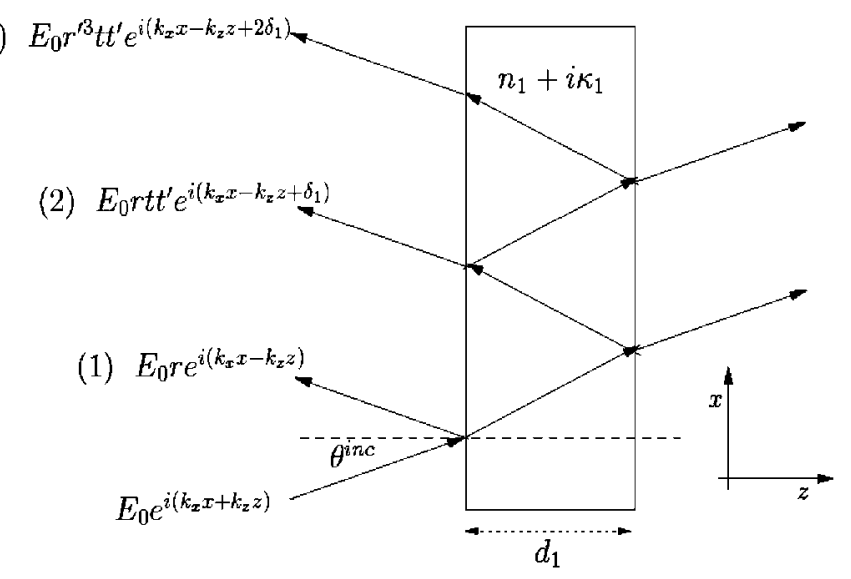

Figure 2. Scattering by a slab.

In the single-layer case (see figure 2) with refractive index $n(z)=n_{1}$ and thickness $d_{1}$, the solution of equation (1) in region 1 under an incident wave in the direction $(\alpha, \beta), u^{\mathrm{inc}}=E_{0} \mathrm{e}^{\mathrm{i}\left(k_{x} x+k_{z} z\right)}=E_{0} \mathrm{e}^{\mathrm{i} k_{0}(\alpha x+\beta z)}$ is given by

$$
u(x, z)=u^{\text {inc }}(x, z)+u^{\text {scatt }}(x, z) \text {, }
$$

where the reflected wave $u^{\text {scatt }}(x, z)$ (that is, the wave scattered by the sample in the backward direction) is given by [18]

$$
u^{\text {scatt }}(x, z)=E_{0} \mathrm{e}^{\mathrm{i}\left(k_{x} x-k_{z} z\right)}\left[r+\frac{r^{\prime} t t^{\prime} \mathrm{e}^{\mathrm{i} \delta_{1}}}{1-r^{\prime 2} \mathrm{e}^{\mathrm{i} \delta_{1}}}\right] .
$$

In equation (2) and in figure 2 the parameters $r, t, r^{\prime}$ and $t^{\prime}$ denote Fresnel coefficients: in the notation of equations (6) and (7) below these are given by $r=r_{0,1}, t=t_{0,1}, r^{\prime}=r_{1,2}=-r_{0,1}$ and $t^{\prime}=t_{1,0}$, with $n_{0}=1, \kappa_{0}=0$, and $n_{1}+\mathrm{i} \kappa_{1}$ equal to the refractive index of the layer. Further, calling $\theta^{\text {trans }}$ the complex transmission angle given by the complex form of Snell's law, $\sin \left(\theta^{\text {inc }}\right) / \sin \left(\theta^{\text {trans }}\right)=n_{1}+\mathrm{i} \kappa_{1}$, the parameter $\delta_{1}$ is given by

$$
\delta_{1}=2 n_{1} d_{1} \cos \left(\theta^{\text {trans }}\right) k_{0} .
$$

If $\kappa_{1}=0$ then $\delta_{1}$ is real, and it equals the optical path-length difference between adjacent rays_-see, e.g., the rays (1) and (2) in figure 2.

It is important for us to note that the scattered wave $u^{\text {scatt }}(x, z)$ may be viewed as the sum of contributions from an infinite number of reflections given by

$$
\begin{aligned}
& E_{1 r}=E_{0} r \mathrm{e}^{\mathrm{i}\left(k_{x} x-k_{z} z\right)}, \\
& E_{2 r}=E_{0} t r^{\prime} t^{\prime} \mathrm{e}^{\mathrm{i} \delta_{1}} \mathrm{e}^{\mathrm{i}\left(k_{x} x-k_{z} z\right)}, \\
& E_{3 r}=E_{0} t r^{\prime 3} t^{\prime} \mathrm{e}^{\mathrm{i} 2 \delta_{1}} \mathrm{e}^{\mathrm{i}\left(k_{x} x-k_{z} z\right)}, \\
& \vdots \\
& E_{N r}=E_{0} t r^{\prime(2 N-3)} t^{\prime} \mathrm{e}^{\mathrm{i}(N-1) \delta_{1}} \mathrm{e}^{\mathrm{i}\left(k_{x} x-k_{z} z\right)} .
\end{aligned}
$$

For a multi-layer film, in turn, multiple reflections occurring at the various interfaces within the sample will contribute to the overall backscattering from the sample. As we will see, only a small number of such interface reflections contribute to the brightness of any one interference 
fringe; for reference, however, we note that the overall field backscattered from the sample, containing contributions from all interface multiple-reflections, is given by

$$
u^{\text {scatt }}(x, z)=E_{0} \widetilde{r}_{0,1} \mathrm{e}^{\mathrm{i}\left(k_{x} x-k_{z} z\right)},
$$

where, calling $\theta_{q}^{\text {trans }}$ the $q$ th transmission angle and $\delta_{q}=2 n_{q} d_{q} \cos \left(\theta_{q}^{\text {trans }}\right) k_{0}$, the generalized reflection coefficients $\tilde{r}_{q, q+1}$ at the interface between region $q$ and region $q+1$ are given by the recurrence relation

$$
\widetilde{r}_{q, q+1}=\frac{r_{q, q+1}+\widetilde{r}_{q+1, q+2} \mathrm{e}^{\mathrm{i} \delta_{q+1}}}{1+r_{q, q+1} \widetilde{r}_{q+1, q+2} \mathrm{e}^{\mathrm{i} \delta_{q+1}}} .
$$

Here $r_{q, q+1}$ are the regular Fresnel reflection coefficient

$$
r_{q, q+1}=\frac{\left(n_{q}+\mathrm{i} \kappa_{q}\right)-\left(n_{q+1}+\mathrm{i} \kappa_{q+1}\right)}{n_{q}+\mathrm{i} \kappa_{q}+n_{q+1}+\mathrm{i} \kappa_{q+1}} .
$$

For future reference we quote here the Fresnel transmission coefficients as well:

$$
t_{q, q+1}=\frac{2\left(n_{q}+\mathrm{i} \kappa_{q}\right)}{n_{q}+\mathrm{i} \kappa_{q}+n_{q+1}+\mathrm{i} \kappa_{q+1}},
$$

see e.g. [17].

In most OCT devices, the incident beams are focused by a lens at a point within the sample, in a setup that gives rise to low numerical apertures. For simplicity and clarity in the exposition, in the remainder of this paper we consider incident fields with normal incidence, that is, incident fields of the form

$$
u^{\mathrm{inc}}(x, z, \omega)=\mathrm{e}^{\mathrm{i} k_{0} z}=\mathrm{e}^{\mathrm{i} \frac{w}{c} z}
$$

it should be clear that an analogous treatment can be given for the case of general incidence. Such general cases have indeed been implemented as part of our direct and inverse-problem solvers; see [19].

\subsection{Coherence}

The OCT technique under consideration is based on use of a Michelson interferometer together with a low-coherence light source, as shown in figure 1. As the sample to be imaged is placed in one arm of the interferometer, the light reflected from the reference mirror and the light backscattered from the sample are combined at the detector; the intensity of the interference fringes that result as the position of the sample is varied is the data from which an image of the interior of the sample is to be obtained.

The total optical intensity received by the detector per unit area is given by [20]

$$
\begin{aligned}
I_{\text {det }} & =\left\langle\left|u^{\text {ref }}(T)+u^{\text {scatt }}(T)\right|^{2}\right\rangle_{T} \\
& =\left\langle\left|u^{\text {ref }}(T)\right|^{2}\right\rangle_{T}+\left\langle\left|u^{\text {scatt }}(T)\right|^{2}\right\rangle_{T}+2 \operatorname{Re}\left\langle\left(u^{\text {ref }}(T)\right)^{*} u^{\text {scatt }}(T)\right\rangle_{T},
\end{aligned}
$$

where ${ }^{*}$ denotes complex conjugate, $u^{\text {ref }}(T)$ and $u^{\text {scatt }}(T)$ represent the fields back-scattered from the reference arm and the sample arm respectively, and where the symbol $\langle\cdots\rangle_{T}$ denotes time averages

$$
\langle G\rangle_{T}=\lim _{T \rightarrow \infty} \frac{1}{T} \int_{-T / 2}^{T / 2} G(s) \mathrm{d} s .
$$

Since the random processes associated with the emission of light are stationary and ergodic $[22,20]$, all the time averages we will encounter can be equated to ensemble averages,

$$
\langle G(t)\rangle_{T}=\langle G(\omega)\rangle_{\Omega}
$$


Here $\langle G(\omega)\rangle_{\Omega}$ denotes ensemble averages over the space $\Omega$ of realizations: denoting by $M$ its probability measure we have

$$
\langle G(\omega)\rangle_{\Omega}=\int_{\Omega} G(\omega) M(\mathrm{~d} \Omega)
$$

In view of ergodicity, in what follows we identify time averages and ensemble averages, and denote either of them by means of the symbol $\langle\cdots\rangle$.

From equation (9) and since the reference intensity stays constant and the sample intensities vary slowly as the sample is moved, the interference fringes that appear in the light-intensity patterns can only result from the term

$$
\Sigma=\left\langle\left(u^{\text {ref }}(T)\right)^{*} u^{\text {scatt }}(T)\right\rangle .
$$

Clearly, $\Sigma$ can be obtained by subtracting from $I_{\text {det }}$ the sum

$$
\left\langle\left|u^{\text {ref }}(T)\right|^{2}\right\rangle+\left\langle\left|u^{\text {scatt }}(T)\right|^{2}\right\rangle .
$$

Each one of the intensities in equation (11) can be measured by closing one arm in the Michelson interferometer; e.g, the intensities $\left\langle\left|u^{\text {scatt }}(T)\right|^{2}\right\rangle$ can be obtained by taking measurements with the reference arm closed.

We now seek to express $\Sigma$ in terms of the statistical properties of the light source. To do this we denote by $u^{\text {src }}(x, z, T)$ and $A(x, z, \omega)$ the field emitted by the source and its Fourier transform with respect to $T$, respectively:

$$
\begin{aligned}
& u^{\mathrm{src}}(x, z, T)=\frac{1}{\sqrt{2 \pi}} \int_{-\infty}^{\infty} A(x, z, \omega) \mathrm{e}^{-\mathrm{i} \omega T} \mathrm{~d} \omega, \\
& A(x, z, \omega)=\frac{1}{\sqrt{2 \pi}} \int_{-\infty}^{\infty} u^{\mathrm{src}}(x, z, T) \mathrm{e}^{\mathrm{i} \omega T} \mathrm{~d} T
\end{aligned}
$$

clearly, for the normal-incidence incident fields we consider (see section 2.1), we have

$$
A(x, z, \omega)=\tilde{A}(\omega) \mathrm{e}^{\mathrm{i} \frac{w}{c} z}, \quad \text { for some function } \tilde{A}(\omega) .
$$

The source beam travels through the source arm of the interferometer and, after passing through the splitter, gives rise to incident fields

$$
u_{r}^{\text {inc }}(z, T)=\frac{1}{2 \sqrt{2 \pi}} \int_{-\infty}^{\infty} \tilde{A}(\omega) \tilde{u}_{r}^{\text {inc }}(z, \omega) \mathrm{e}^{-\mathrm{i} \omega T} \mathrm{~d} \omega
$$

and

$$
u_{s}^{\text {inc }}(z, T)=\frac{1}{2 \sqrt{2 \pi}} \int_{-\infty}^{\infty} \tilde{A}(\omega) \tilde{u}_{s}^{\text {inc }}(z, \omega) \mathrm{e}^{-\mathrm{i} \omega t} \mathrm{~d} \omega
$$

on the reference (i.e. mirror) and sample arms, respectively, where ${\tilde{u_{r}}}^{\text {inc }}(z, \omega)$ and ${\tilde{u_{s}}}^{\text {inc }}(z, \omega)$ denote plane waves

$$
\tilde{u}_{r}^{\text {inc }}(z, \omega)=\tilde{u}_{s}^{\text {inc }}(z, \omega)=\mathrm{e}^{\mathrm{i} \frac{\omega}{c}(z+D)} .
$$

Note that distances along the mirror and sample arms are both denoted by the same symbol $z$. Further, note the factors of $\frac{1}{2}$ in these integrals, which account for our assumed half-and-half split caused by the beam splitter.

The reference and sample backscattered fields received by the detector are thus given by

and

$$
u^{\mathrm{ref}}(T)=\frac{1}{4 \sqrt{2 \pi}} \int_{-\infty}^{\infty} \tilde{A}(\omega) \tilde{u}^{\mathrm{ref}}(\omega) \mathrm{e}^{-\mathrm{i} \omega T} \mathrm{~d} \omega,
$$

$$
u^{\text {scatt }}(T)=\frac{1}{4 \sqrt{2 \pi}} \int_{-\infty}^{\infty} \tilde{A}(\omega) \tilde{u}^{\text {scatt }}(\omega) \mathrm{e}^{-\mathrm{i} \omega T} \mathrm{~d} \omega
$$


where $\tilde{u}^{\text {ref }}(\omega)=\mathrm{e}^{-\mathrm{i} \frac{\omega}{c} 2(D+\eta)}$ and $\tilde{u}^{\text {scatt }}(\omega)$ are the values at the detector of the field backscattered by the mirror and the sample, respectively. Note the additional factors of $\frac{1}{2}$ in these formulae which account, once again, for the splitting of beams by the splitter.

Substituting equations (16) and (17) into (10) we obtain

$$
\Sigma=\frac{1}{32 \pi} \int_{-\infty}^{\infty} \int_{-\infty}^{\infty}\left\langle\tilde{A}^{*}(\omega) \tilde{A}\left(\omega^{\prime}\right)\right\rangle\left(\tilde{u}^{\mathrm{ref}}(\omega)\right)^{*} \tilde{u}^{\text {scatt }}\left(\omega^{\prime}\right) \mathrm{e}^{\mathrm{i} \omega T} \mathrm{e}^{-\mathrm{i} \omega^{\prime} T} \mathrm{~d} \omega \mathrm{d} \omega^{\prime}
$$

But we have

$$
\begin{aligned}
\left\langle\tilde{A}^{*}(\omega) \tilde{A}\left(\omega^{\prime}\right)\right\rangle & =\int_{\Omega} \tilde{A}^{*}(\omega) \tilde{A}\left(\omega^{\prime}\right) M(\mathrm{~d} \Omega) \\
& =\frac{1}{2 \pi} \int_{-\infty}^{\infty} \int_{-\infty}^{\infty}\left\langle u^{*}(T) u\left(T^{\prime}\right)\right\rangle \mathrm{e}^{\mathrm{i} \omega T} \mathrm{e}^{-\mathrm{i} \omega^{\prime} T^{\prime}} \mathrm{d} T \mathrm{~d} T^{\prime} \\
& =\frac{1}{2 \pi} \int_{-\infty}^{\infty} \int_{-\infty}^{\infty} \Gamma\left(T-T^{\prime}\right) \mathrm{e}^{\mathrm{i} \omega T} \mathrm{e}^{-\mathrm{i} \omega^{\prime} T^{\prime}} \mathrm{d} T \mathrm{~d} T^{\prime} \\
& =\frac{1}{2 \pi} \int_{-\infty}^{\infty} \Gamma(\tau) \mathrm{e}^{\mathrm{i} \omega \tau} \mathrm{d} \tau \int_{-\infty}^{\infty} \mathrm{e}^{\mathrm{i}\left(\omega-\omega^{\prime}\right) T} \mathrm{~d} T \\
& =S(\omega) \delta\left(\omega-\omega^{\prime}\right),
\end{aligned}
$$

where $\tau=T-T^{\prime}$ and $\Gamma(\tau)=\left\langle u^{*}(T-\tau), u(T)\right\rangle$ is the self-coherence function, and where its Fourier transform $S(\omega)$ is the power spectral density of the optical field, respectively. It follows that $\Sigma$ can be expressed in terms of the power spectral density $S(\omega)$

$$
\Sigma=\frac{1}{16 \sqrt{2 \pi}} \int_{-\infty}^{\infty} S(\omega)\left(\tilde{u}^{\mathrm{ref}}(\omega)\right)^{*} \tilde{u}^{\text {scatt }}(\omega) \mathrm{d} \omega,
$$

which gives us the required expression for $\Sigma$ in terms of the statistical properties of the light source and the refractive properties of the sample under pure plane waves of form (15).

As is commonly done, in our work we will assume a Gaussian power spectral density of the form

$$
S(\omega)=\frac{4 \sqrt{\log (2) \pi}}{\Delta \omega} \mathrm{e}^{-\left(2 \sqrt{\left.\log (2) \frac{\omega-\bar{\omega}}{\Delta \omega}\right)^{2}}\right.}
$$

whose complex degree of coherence is given by

$$
\gamma(\tau)=\mathrm{e}^{-\left(\frac{\Delta \omega \tau}{4 \sqrt{\log (2)}}\right)^{2}} \mathrm{e}^{-\mathrm{i} \bar{\omega} \tau}
$$

Here, calling $\lambda$ and $\Delta \lambda$ the centre wavelength and the full-width at half-maximum (FWHM) respectively, $\bar{w}=2 \pi c / \lambda$ is the central angular frequency and $\Delta \omega=2 \pi c \Delta \lambda / \lambda^{2}$ is the width of the spectrum line between the half-power points [20]. Following [21], the coherence length of the source is given by

$$
l_{c}=\frac{2 \pi c}{\Delta \omega}=\frac{\lambda^{2}}{\Delta \lambda}
$$

\subsection{Data collection and the OCT inverse problem}

For definiteness, and in general accordance with methods reported in the recent literature, we assume OCT data are collected as follows. At first the sample and mirror are placed in such a way that the sample's leftmost boundary lies at the point $\xi=\xi_{0}$ and the mirror is at a distance $\zeta=\xi_{0}$, see figure 3. Next, the sequence of intensity-fringe values $\Sigma$ that occur as the sample is relocated at the sequence of positions $\xi=\xi_{0}-j \varepsilon$ is obtained-where $\varepsilon$ is a step-size (which, 


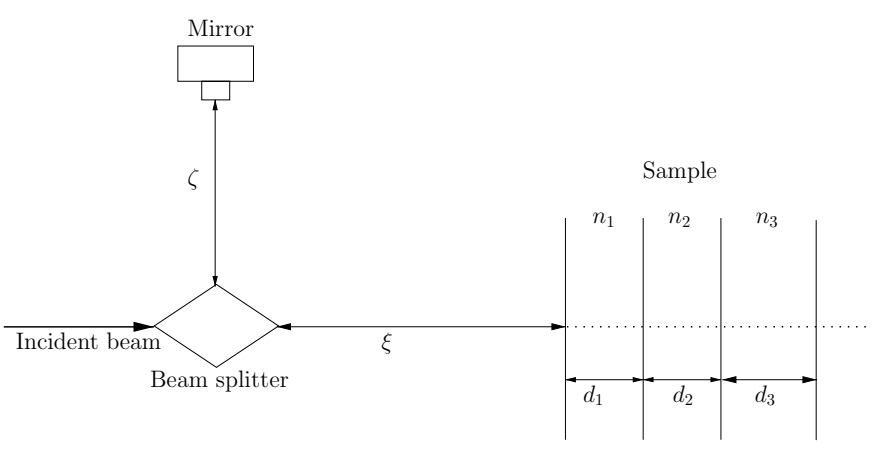

Figure 3. Scanning process starts with $\xi=\xi_{0}$. Throughout the scan $\zeta$ is fixed at the value $\zeta=\xi_{0}$.
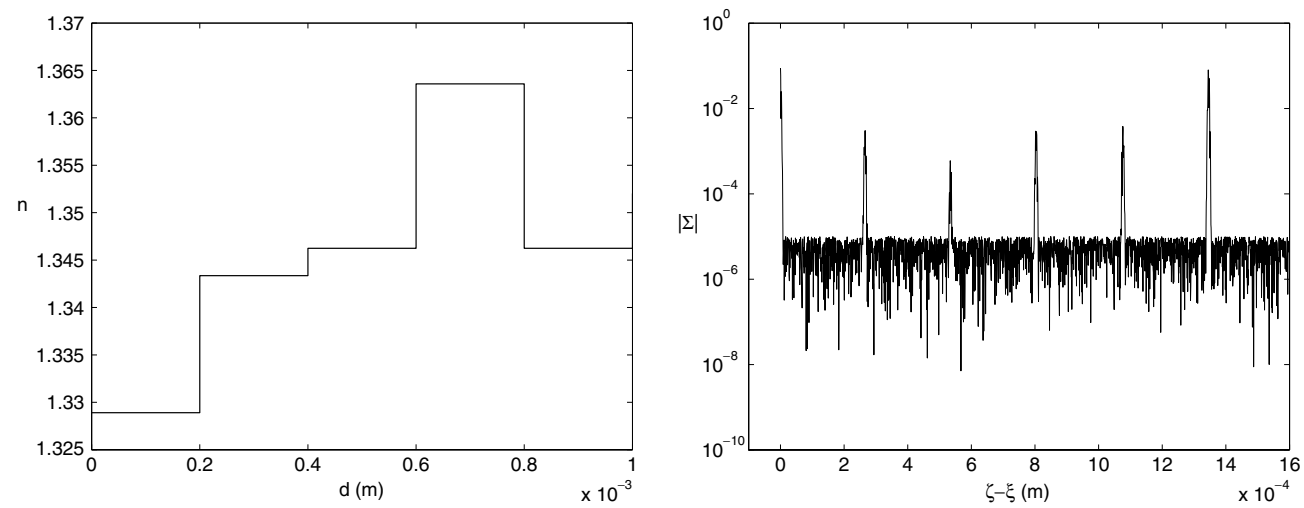

Figure 4. Left: refractive index distribution for a 5-layer structure. Right: data obtained as a result of the collection procedure detailed in section 2.3 (simulated).

as discussed below, should be chosen appropriately), and where $j$ ranges from 1 to a number $N^{\text {meas }}$ of measurements. The right portions of figures 4 and 5 , for example, show the total fringe intensity $|\Sigma|$ (as functions of $\xi$ ) arising from this procedure for the layered structures depicted on the left-hand sides of those figures. (In these and all the subsequent numerical simulations in this paper we have added to the exact computed correlation $\Sigma$ random noise of the order of $10^{-4}$ relative to the intensity signal of the first reflection - which is itself of the order of $10^{-1}$ - so that the added noise, equal to $10^{-5}$ times a random number between -1 and 1 , is of the order $10^{-5}$. This noise level is much larger than the various noise bars reported in the literature [15, 23-26].) The marked spikes in the right graph in figure 4 correspond to light scattered by the interfaces: from left to right the spikes correspond to the interfaces between air and the first layer, between the first two layers in the sample, and so on, and the last spike corresponds to reflection between the last interface and air.

In figures 6 and 7 we show blow-ups of the two first spikes in figure 4 right, respectively; we see that these spikes have a definite structure. Our methods utilize coarse samples of those spike structures (e.g., the black circles in figure 7 right) to determine the refractive index distributions within the sample. It may be surprising at first that such coarse sampling of these spikes suffice to obtain width and refractive index values within the sample. However, this is not unreasonable: although the peak is indeed under-sampled (i.e., an accurate rendering of the peak cannot be produced on the basis of such sampling alone), the least-squares problem 

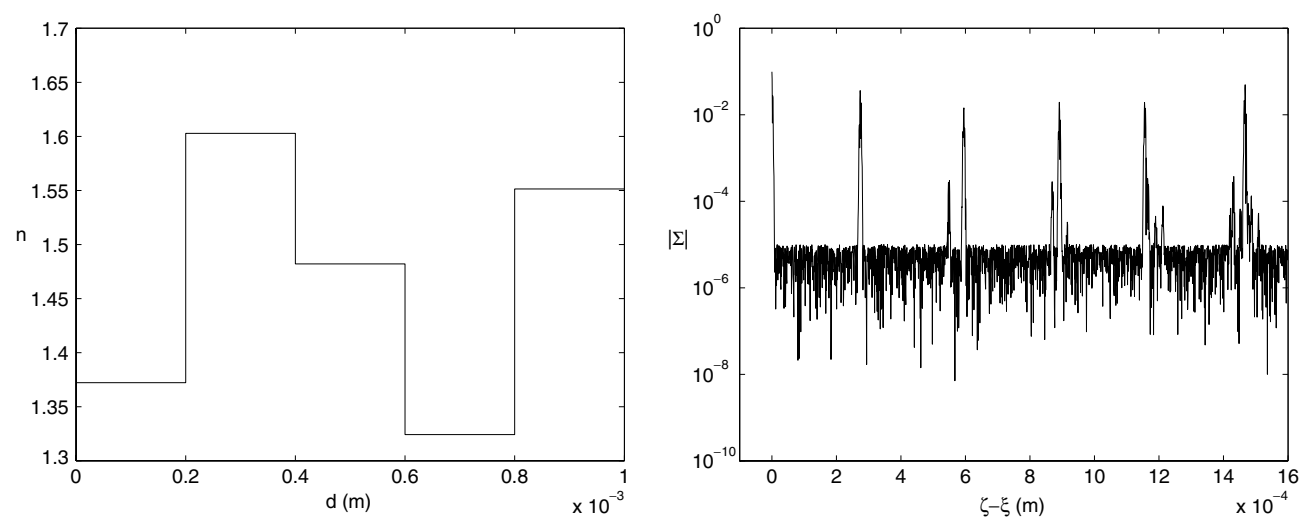

Figure 5. Left: refractive-index distribution for a 5-layer structure-with higher refractiveindex contrast than that of figure 4. Right: data obtained as a result of the procedure detailed in section 2.3 (simulated). Note the appearance of spikes that do not correspond to interfaces between layers.
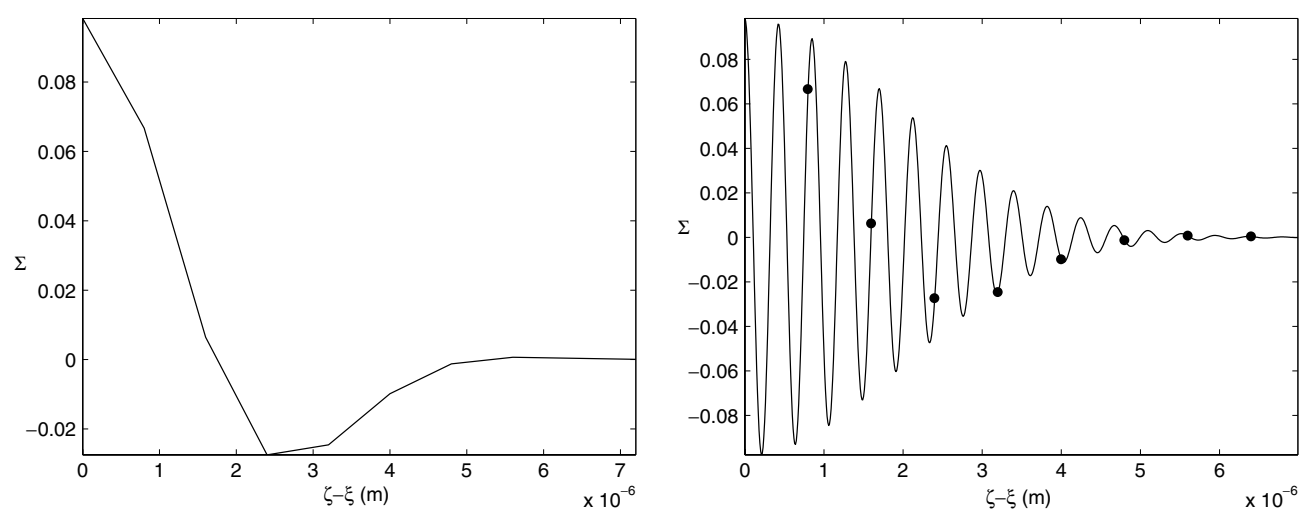

Figure 6. Left: coarsely sampled first spike in figure 4. Right: finely sampled first spike, with black circles showing the coarse samples displayed on the left.

discussed in section 3 for determination of width and refractive index, which results from such measurements, is actually over-determined, since it seeks to produce only two values, the current width and refractive index, from a relatively large number of measurements, e.g., as many as sampling points in figures 6 and 7.

(As mentioned above, it is important to select of the parameter $\varepsilon$ appropriately. The value $\varepsilon=\lambda$, for example, would lead to highly aliased measurements such as those shown in 'o-plus' circles in figure 7. Clearly, the o-plus data-points contain information of poor quality, since they lie close to the noise floor and are thus significantly polluted by it. Solving the inverse problem with such polluted data is difficult, and may even lead to incorrect solutions. We have found that values of $\varepsilon$ close to but slightly different from the laser centre wavelength lead to good fringe sampling and are therefore adequate.)

Our OCT inverse solver sequentially determines interference spikes which are most relevant to the solution of the inverse problem, and uses the information contained in them to determine refractive index values and layer widths, as explained in what follows. Throughout 

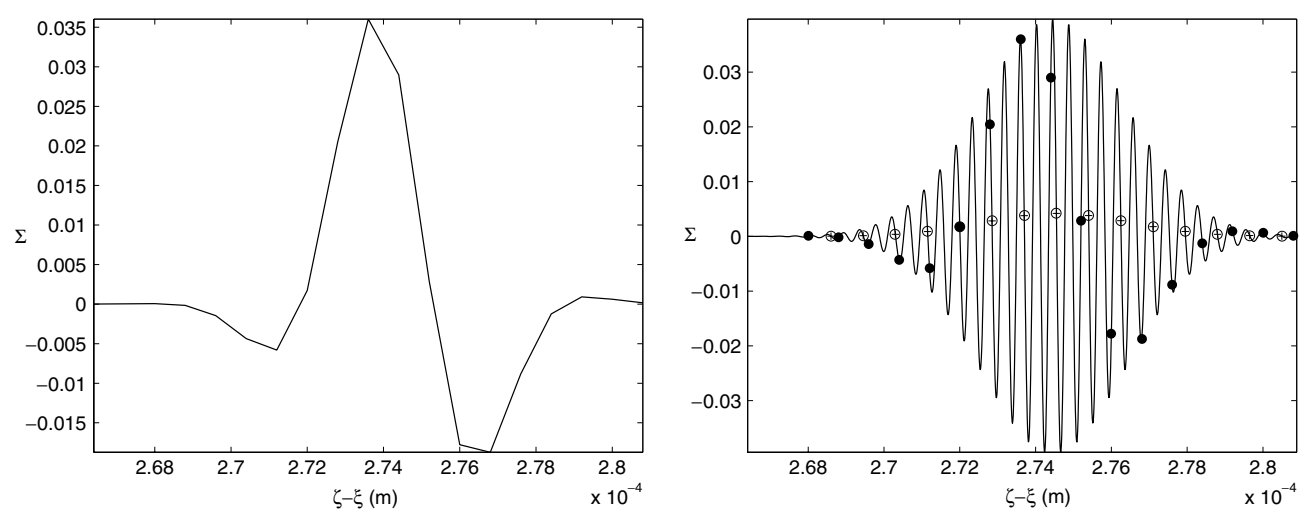

Figure 7. Left: coarsely sampled second spike in figure 4. Right: finely sampled second spike, with black circles showing the coarse samples displayed on the left, and with 'o-plus' circles showing coarse 'aliased' samples providing data of very small absolute value-which is prone to be degraded by noise.

this paper we assume all layers widths are large compared to the coherence length of the source.

Determination of the refractive index of the first layer. We begin by noting that, provided the thickness of the first layer is sufficiently large compared with the coherence length of the source, as we have assumed, the first group of data measurements (the left spike in figure 4 right) is only determined by the refractive index of the first layer. Indeed, the second and subsequent interfaces do not contribute significantly to the first fringe pattern, since the rays reflected by them travel a much longer optical path than the rays reflected by the mirror. More precisely, in view of the exponential decay of the coherence function $\gamma$, up to exponentially small errors the reflection from the second and subsequent interfaces do not contribute to the first spike; in view of our standing assumption that the layer widths are much larger than the coherence length, such exponentially small contributions are neglected. The problem of evaluation of the refractive index of the first layer thus reduces to one that may be treated by the least-squares method described in section 3 .

Determination of widths of the first layer and the refractive index of the second layer. Once the refractive index of the first layer has been obtained, the algorithm proceeds to determine its thickness together with the refractive index of the second layer. To do this the algorithm utilizes the second spike (depicted in figure 7 left) which arises, mainly, as a result of reflections from the second interface. The spikes themselves are characterized as groups of values of $|\Sigma|$ above a certain threshold $\Sigma^{\text {thresh }}$; in most of our examples we have used threshold value $\Sigma^{\text {thresh }}$ equal to the noise floor, see section 2.3. Again, in view of the exponential decay of the coherence function $\gamma$, up to exponentially small errors the reflection from the third and subsequent interfaces do not contribute to the second spike. At this stage we use a least-square method together with the minimization algorithm detailed in section 3 below to produce the desired refractive index $n_{2}$ and thickness $d_{1}$.

Determination of widths of the qth layer and the refractive index of the $(q+1)$ th layer. A similar procedure is utilized to determine the thicknesses and refractive indexes of subsequent layers from corresponding spikes. As discussed in what follows, not all spikes are associated with actual interfaces: those that are will be referred to as primary spikes. To introduce this concept, we first give a precise definition of the notion of spike. 
(i) A spike is a group of consecutive values of $|\Sigma|$ above the threshold $\Sigma^{\text {thresh }}$ (consecutive perhaps with the exceptions described in points (ii) and (iii) below). A group of large values of $|\Sigma|$ arising from an interface may occasionally enclose a value of less than $\Sigma^{\text {thresh }}$, which would unduly split the spike into two. To avoid this we require that:

(ii) For two set of values to be considered part of different spikes, they must be separated by a certain minimum number $n_{\text {low }}^{\text {min }}$ of consecutive low measurement values between them. In all our examples we have chosen $n_{\text {low }}^{\min }=6$. (Here we see an example of the way in which the coherence length determines the resolution of the method: the separation by a number $n_{\text {low }}^{\text {min }}$ of measurements between two measurement peaks can only exist if the coherence length is smaller than half the width of the layer.) If two sequences of values of $\Sigma$ above $\Sigma^{\text {thresh }}$ are separated by a number less than or equal to $n_{\text {low }}^{\min }$ of values below $\Sigma^{\text {thresh }}$, the two groups are considered to be part of the same spike.

(iii) Similarly, a spike must contain a certain minimum number $n_{\text {high }}^{\min }$ of consecutive high values of $\Sigma$; in all of our examples we have chosen $n_{\text {high }}^{\min }=4$. If a sequence of high values contains fewer than $n_{\text {high }}^{\min }$ points then it is not considered to be a spike.

(iv) Primary spikes. A spike, as defined by points (i)-(iii) above may result solely from combinations of multiple reflections from various interfaces and thus, it may not identify any one interface; see figure 5. To ensure a spike we use identifies a true interface we must evaluate the contribution $\Sigma^{\text {prev }}$ to the correlation $\Sigma$ for the current position of the sample which arise from reflections from all previous interfaces. If the mean-square norm of the difference between $\Sigma^{\text {prev }}$ and the measured $\Sigma$ for values of $\xi$ associated with a given spike is less than the $\Sigma^{\text {thresh }}$ then the spike under consideration does not identify a new interface, and it is thus considered to be a secondary spike; otherwise, the spike does identify a new interface and it is called a primary spike.

Our algorithm determines sequentially widths and refractive indexes as follows: assuming the first $(q-1)$ widths and the first $q$ refractive indexes have been obtained, the algorithm first identifies the $(q+1)$ th primary spike-by carrying the procedures implicit in our definition of that concept - and then, using that primary spike together with the minimization procedure described in section 3 , it produces $d_{q}$ and $n_{q+1}$. Inductively, the algorithm produces primary spikes and corresponding pairs of refractive indexes and widths until all available spikes are used, and the desired refractive index map of the sample is obtained.

\subsection{Fast evaluation of the function $\Sigma$}

As mentioned above, our numerical method uses measured values of $\Sigma$ to determine the distributions of refractive index within the sample, and, to do this, it requires an effective method for evaluation of $\Sigma$ for a prescribed refractive index distribution. It is easy to see, however, that the numerical evaluation of $\Sigma$ through direct integration of expression (19) for each mirror and sample position would lead to inordinately long computing times. Fortunately, the low-coherence property of the light allows us to obtain $\Sigma$ in an extremely simple and fast manner, as explained in what follows.

To motivate the introduction of our fast evaluation algorithm, we begin by considering a configuration for which the sample consists of a single homogeneous slab under the normally incident wave (8); the implications of this analysis on the multi-layer case are described later in this section. Under the present single-layer, normal-incidence assumptions, it follows that the backscattering received from the sample at the detector for a fixed mirror position $\zeta$ depends only on frequency and the position $\xi$ of the sample:

$$
\tilde{u}^{\text {scatt }}(\xi, \omega)=\tilde{u}^{\text {scatt }}(\xi, \omega)=\frac{1}{4} \mathrm{e}^{\mathrm{i} k(D+\eta+2 \xi)+\pi \mathrm{i}}\left[r+\frac{r^{\prime} t t^{\prime} \mathrm{e}^{\mathrm{i} \delta_{1}}}{1-r^{\prime 2} \mathrm{e}^{\mathrm{i} \delta_{1}}}\right],
$$


where $\eta$ is defined in figure 1 , and $r=r_{0,1}, t=t_{0,1}, r^{\prime}=r_{1,2}=-r_{0,1}, t^{\prime}=t_{1,0}$, and $\delta_{1}$ are the Fresnel coefficients defined in section 2.1. For the reflection from the mirror, on the other hand, we have

$$
\tilde{u}^{\mathrm{ref}}(\omega)=\tilde{u}^{\mathrm{ref}}(\omega)=\frac{1}{4} \mathrm{e}^{\mathrm{i} k(D+\eta+2 \zeta)+2 \pi \mathrm{i}}=\frac{1}{4} \mathrm{e}^{\mathrm{i} k(D+\eta+2 \zeta)},
$$

where $D, \zeta$ and $\xi$ are defined in figure 1 . The $2 \pi \mathrm{i}$ constant in the first exponent of equation (22) accounts for two phase jumps of $\pi \mathrm{i}$, which arise as the beam is reflected by the splitter mirror and the reference mirror, respectively. The factor $\frac{1}{4}$, in turn, is due to the fact that light passes twice through the splitter.

Denoting

$$
\tau=2(\zeta-\xi) / c
$$

function (19) for the present single-layer configuration is given by

$$
\Sigma(\xi)=-\frac{1}{16 \sqrt{2 \pi}} \int_{-\infty}^{\infty} S(\omega)\left[r+\frac{r^{\prime} t t^{\prime} \mathrm{e}^{\mathrm{i} \delta_{1}}}{1-r^{\prime 2} \mathrm{e}^{\mathrm{i} \delta_{1}}}\right] \mathrm{e}^{-\mathrm{i} \omega \tau} \mathrm{d} \omega,
$$

or, in view of equation (3)

$$
\Sigma(\xi)=-\frac{1}{16 \sqrt{2 \pi}} \int_{-\infty}^{\infty} S(\omega)\left[r+\frac{r^{\prime} t t^{\prime} \mathrm{e}^{\mathrm{i} \omega T_{1}^{\text {delay }}}}{1-r^{\prime 2} \mathrm{e}^{\mathrm{i} \omega T_{1}^{\text {delay }}}}\right] \mathrm{e}^{-\mathrm{i} \omega \tau} \mathrm{d} \omega,
$$

where $T_{1}^{\text {delay }}=\delta_{1} / \omega=2 n_{1} d_{1} / c$; more generally we define

$$
T_{q}^{\text {delay }}=2 n_{q} d_{q} / c \text {. }
$$

Note that, under our standing assumption that $\zeta$ is constant, the correlation $\Sigma$ is a function of $\xi$ only: $\Sigma=\Sigma(\xi)$; see equation (19). Clearly, the integrand in equation (24) is highly oscillatory, and, as it happens, direct evaluation of that integral via resolution of that oscillatory behaviour requires prohibitively long computing times.

To simplify the evaluation of the integral in equation (24) we use a series expansion of the bracketed term in the integrand and obtain

$$
\begin{gathered}
\Sigma(\xi)=-\frac{1}{16 \sqrt{2 \pi}}\left[r \int_{-\infty}^{\infty} S(\omega) \mathrm{e}^{-\mathrm{i} \omega \tau} \mathrm{d} \omega+\left(r^{\prime} t t^{\prime}\right) \int_{-\infty}^{\infty} S(\omega) \mathrm{e}^{\mathrm{i} \omega T_{1}^{\text {delay }}} \mathrm{e}^{-\mathrm{i} \omega \tau} d \omega\right] \\
-\frac{1}{16 \sqrt{2 \pi}}\left(r^{\prime 3} t t^{\prime}\right) \int_{-\infty}^{\infty} S(\omega) \mathrm{e}^{\mathrm{i} 2 \omega T_{1}^{\text {delay }}} \mathrm{e}^{-\mathrm{i} \omega \tau} \mathrm{d} \omega+\cdots \\
-\frac{1}{16 \sqrt{2 \pi}}\left(r^{\prime(2 N-3)} t t^{\prime}\right) \int_{-\infty}^{\infty} S(\omega) \mathrm{e}^{\mathrm{i}(N-1) \omega T_{1}^{\text {delay }}} \mathrm{e}^{-\mathrm{i} \omega \tau} \mathrm{d} \omega+\cdots
\end{gathered}
$$

It follows that,

$$
\begin{gathered}
\Sigma(\xi)=-\frac{\sqrt{2 \pi}}{16}\left[r \gamma(\tau)+\left(r^{\prime} t t^{\prime}\right) \gamma\left(\tau-T_{1}^{\text {delay }}\right)\right]-\frac{\sqrt{2 \pi}}{16}\left(r^{\prime 3} t t^{\prime}\right) \gamma\left(\tau-2 T_{1}^{\text {delay }}\right)+\cdots \\
-\frac{\sqrt{2 \pi}}{16}\left(r^{\prime(2 N-3)} t t^{\prime}\right) \gamma\left(\tau-(N-1) T_{1}^{\text {delay }}\right)+\cdots,
\end{gathered}
$$

where $\gamma(\tau)$ is the complex degree of coherence of the source

$$
\gamma(x)=\frac{1}{2 \pi} \int_{-\infty}^{\infty} S(\omega) \mathrm{e}^{-\mathrm{i} \omega x} \mathrm{~d} \omega .
$$

Series (26) converges extremely fast. To appreciate the physical significance of this expression, let us denote by $j_{0}$ the value of $j$ for which $\tau-j T_{1}^{\text {delay }}$ is closest to zero, and let us note that the $j$ th term in the series accounts for contributions produced by light backscattered after $j$ 
Table 1. The functions $\Sigma(\xi)$, calculated by direct evaluation of integral (24), and $\Sigma^{\text {trunc }}(\xi)$, calculated from a truncated version of equation (26), with truncations as defined in the text. Here $n_{1}=1.4$ and $n_{2}=1.6 ; \tau=2(\zeta-\xi) / c$.

\begin{tabular}{lll}
\hline$\xi$ & $\Sigma(\xi)$ & $\Sigma^{\text {trunc }}(\xi),(C=-1 / 16 \sqrt{2 \pi})$ \\
\hline$\xi=\zeta$ & 0.0522212 & $C r_{0,1} \gamma(\tau)=0.0522214$ \\
$\xi=\zeta-d_{1}^{\text {opt }}$ & 0.0203082 & $C r_{1,2} t_{0,1} t_{1,0} \gamma\left(\tau-d_{1}^{\text {opt }} / c\right)=0.0203083$ \\
$\xi=\zeta-\left(d_{1}^{\text {opt }}+d_{2}^{\text {opt }}\right)$ & -0.0699794 & $C r_{2,3} t_{0,1} t_{1,0} t_{1,2} t_{2,1} \gamma\left(\tau-2\left(d_{1}^{\text {opt }}+d_{2}^{\text {opt }}\right) / c\right)=-0.0699856$ \\
\hline
\end{tabular}

bounces within the layer. The factor $\gamma\left(\tau-j T_{1}^{\text {delay }}\right)$, which takes smaller and smaller values as $j$ departs farther and farther away from $j_{0}$, accounts for the reduced contributions to the function $\Sigma$ that occur as a result of the loss of coherence associated with departures of $j$ from $j_{0}$. Analogous (albeit more complex) ray expansions can be obtained for any given number of layers and for arbitrary incidences.

To visualize the importance of these rapidly convergent ray expansions in our context we first analyse the convergence of series (26) for a few significant examples. In our first example the sample is a structure consisting of two layers of width $d_{1}=d_{2}=10 \mu \mathrm{m}$ containing materials of refractive indexes $n_{1}=1.4$ and $n_{2}=1.6$, respectively. We denote by $d_{1}^{\text {opt }}=n_{1} d_{1}$ and $d_{2}^{\text {opt }}=n_{2} d_{2}$ their respective optical path lengths. In what follows the symbol $\Sigma^{\text {trunc }}(\xi)$ denotes various approximations of the function $\Sigma(\xi)$; the reflected waves to be included in $\Sigma^{\text {trunc }}$ which will vary through this discussion, should be clear from the context.

Table 1 contains values of the function $\Sigma$ and various truncations $\Sigma^{\text {trunc }}$. To define the relevant contributions to each one of these truncations we note that, for the present two-layer structure we have

$$
\begin{aligned}
& \tilde{u}^{\text {scatt }}(\omega)=-\frac{1}{4} \tilde{r}_{0,1} \mathrm{e}^{\mathrm{i} k(D+\eta+2 \xi)}, \\
& \tilde{u}^{\mathrm{ref}}(\omega)=\frac{1}{4} \mathrm{e}^{\mathrm{i} k(D+\eta+2 \zeta)},
\end{aligned}
$$

where $\tilde{r}_{0,1}$ is given by equation (5), so that, in this case, equation (19) becomes

$$
\Sigma(\xi)=-\frac{1}{16 \sqrt{2 \pi}} \int_{-\infty}^{\infty} S(\omega) \tilde{r}_{0,1} \mathrm{e}^{-\mathrm{i} \omega \tau} \mathrm{d} \omega,
$$

with $\tau(\xi)$ given by equation (23). This function may be viewed as a sum of contributions resulting from reflections of rays on the various interfaces, which are given, in turn, by the coefficients $r_{q, q+1}$ and $t_{q, q+1}$ defined in equations (6) and (7).

In rows 2,3 and 4 of table 1 , the truncated function $\Sigma^{\text {trunc }}$ includes contributions as follows. Row 2: the ray reflected into the detector directly from the first interface (with a contribution of $\left.-1 / 16 \sqrt{2 \pi} r_{0,1} \gamma(0)\right)$, see figure 8 , top left. Row 3: the portion of the ray reflected at the second interface which is transmitted back into the detector through the first interface (with a contribution of $-1 / 16 \sqrt{2 \pi} r_{1,2} t_{0,1} t_{1,0} \gamma\left(\tau-d_{1}^{\text {opt }} / c\right)$ ), see figure 8 , top right and; row 4: the portion of the ray reflected at the third interface which then impinges on the detector after having been transmitted by both the second and first interfaces (with a contribution of $\left.-1 / 16 \sqrt{2 \pi} r_{2,3} t_{0,1} t_{1,0} t_{1,2} t_{2,1} \gamma\left(\tau-2\left(d_{1}^{\text {opt }}+d_{2}^{\text {opt }}\right) / c\right)\right)$, see figure 8 , bottom left.

The agreement between the exact (as calculated by direct evaluation of integral (24)) and approximate expressions for $\Sigma$ is excellent and, clearly, the approximate expression is much easier to evaluate: in every case we just need one term of the series to obtain the value of integral (27) with an absolute error of the order of $10^{-5}$. In the last row we find the lowest accuracy; this approximation can be improved by adding the contribution of an additional reflection-so as to account for a multiple reflection within the first layer, see figure 8 , bottom 

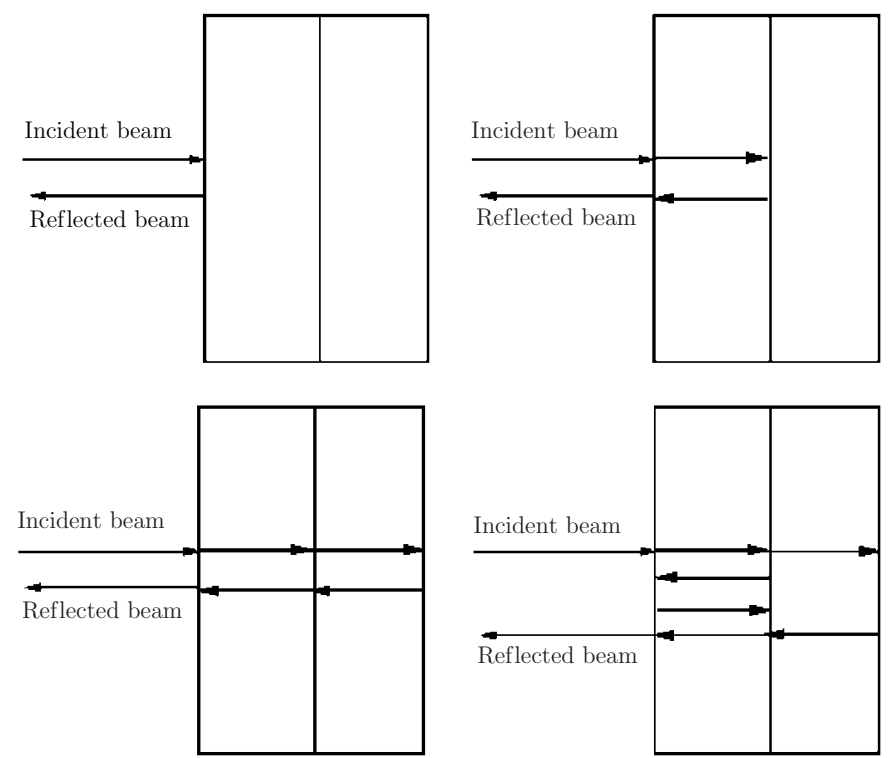

Figure 8. Schematic display of multiple-scattered rays considered in table 1. Left top: first row in table 1. Right top: second row in table 1. Left bottom: third row in table 1. Right bottom: the correction term of equation (28), $\frac{\sqrt{2 \pi}}{16}\left(r_{0,1} r_{1,2}^{2} t_{0,1} t_{1,0} \gamma\left(\tau-4 d_{1}^{\mathrm{opt}} / \omega\right)\right)$.

right. The resulting improved approximation is

$$
\begin{aligned}
\Sigma^{\text {trunc }}=-\frac{\sqrt{2 \pi}}{16} & \left(r_{2,3} t_{0,1} t_{1,0} t_{1,2} t_{2,1} \gamma\left(\tau-2\left(d_{1}^{\mathrm{opt}}+d_{2}^{\mathrm{opt}}\right) / c\right)\right) \\
& +\frac{\sqrt{2 \pi}}{16}\left(r_{0,1} r_{1,2}^{2} t_{0,1} t_{1,0} \gamma\left(\tau-4 d_{1}^{\mathrm{opt}} / \omega\right)\right)=-0.0699794
\end{aligned}
$$

For a multi-layer structure the contribution of multi-reflections becomes more and more important as the number of layers increases. In what follows we show that a ray expansion is still possible even for samples containing a large number of layers.

To do this we decompose the backscattering from the sample as a sum of rays, each one of which results from finitely many interface reflections, and consider associated approximations to the correlation $\Sigma$. The simplest such approximation is $\Sigma_{1}(\xi)$, which we define as the contribution to the total correlation $\Sigma(\xi)$ that results as, amongst the rays that give rise to coherent interference (i.e., those for which $\gamma$ is not small), all rays that undergo more than one interface reflection are neglected. To obtain $\Sigma_{1}(\xi)$ we proceed as follows: given the position $\xi$ of the sample we compute $\tau=2(\zeta-\xi) / c$ and determine the value of $j$ such that the optical path between the 0 th interface and the $j$ th interface is as close as possible to $\zeta-\xi$; in other words

$$
j \text { minimizes }\left|\tau-\sum_{q=1}^{j-1} T_{q}^{\text {delay }}\right| \text { amongst all } j \text { with } 1 \leqslant j \leqslant N^{\text {layers }},
$$

where $T_{q}^{\text {delay }}$ is given by equation (25). Then, $\Sigma_{1}(\xi)$ is given by

$$
\Sigma_{1}(\xi)=-\frac{\sqrt{2 \pi}}{8} \operatorname{Re}\left(\bar{r}_{j-1, j} \gamma\left(\tau-\sum_{s=1}^{j-1} T_{s}^{\text {delay }}\right)\right)
$$



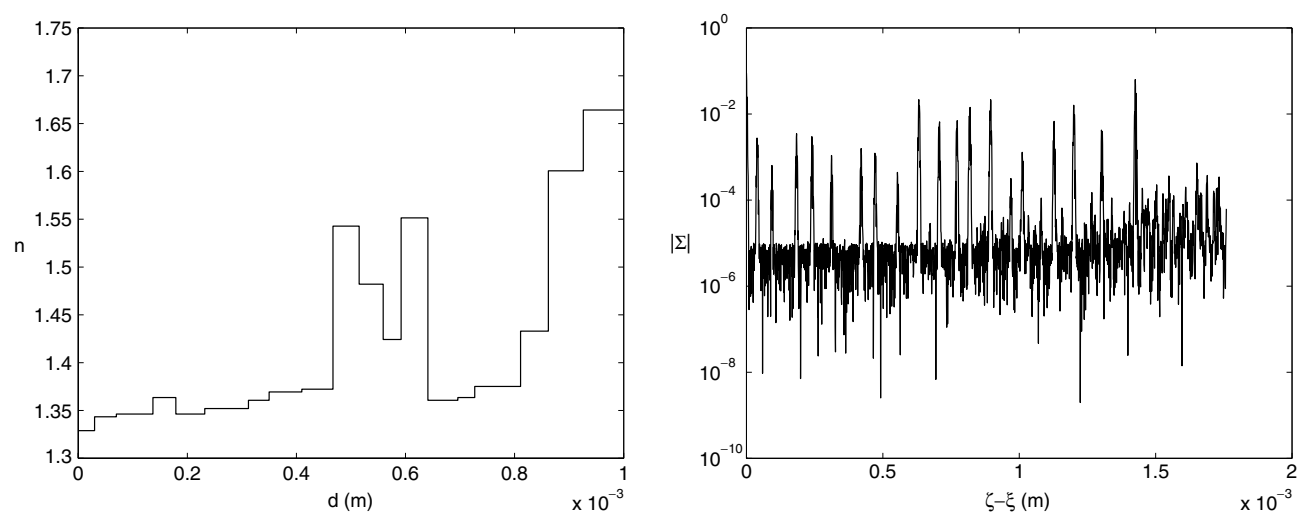

Figure 9. Left: refractive index distribution along a multi-layer structure of thickness $1 \mathrm{~mm}$. Right: display of the corresponding function $|\Sigma(\xi)|$, as defined in equation (19), evaluated at 2200 sample positions $\xi$.

where $\bar{r}$ is given by

$$
\bar{r}_{j-1, j}=r_{j-1, j} \prod_{q=1}^{j-1} t_{q-1, q} t_{q, q-1} .
$$

This expression for $\Sigma_{1}(\xi)$ thus depends on one reflection coefficient, $r_{j-1, j}$, and two transmission coefficients for each one of first $j-1$ interfaces; it is easy to check that, as claimed, $\Sigma_{1}(\xi)$ is the approximation to the total correlation $\Sigma(\xi)$ that results by neglecting multiple reflected rays.

The quantity $\Sigma_{1}$ is usually a poor approximation to $\Sigma$. To improve this approximation we define

$$
\Sigma_{2}(\xi)=\Sigma_{1}(\xi)+\Sigma_{c}(\xi)
$$

where $\Sigma_{c}$ is a correction term including a finite number of multiple reflections needed to reduce the truncation error to a prescribed level which, typically we take to equal the noise floor. In our numerical examples, for instance, we assume a noise floor of the order of $10^{-4}$ relative to the intensity signal of the first reflection-which is itself proportional to $r_{0,1}$ - and consider refractive index values for which the quantities $r_{j-1, j}$ are of the order of $10^{-1}$ for $1 \leqslant j \leqslant N^{\text {layers }}$. In those cases we have $r_{j-1, j}^{5} \approx O$ (noise floor) and thus, to compute $\Sigma_{c}$ to within an error of the order of the prescribed noise floor we need only take into account the reflections involving, at most, three reflections coefficients - thus neglecting multiple-scattered rays containing five or more reflections. (Clearly the number of reflections is necessarily odd.) Of course, the number of reflections needed to achieve a desired error depends on the specific values of the parameters under consideration, and those given above are meant as an illustration only.

To compute $\Sigma_{c}$ under the conditions described in the previous paragraph, we need to add contributions from all the rays which are reflected at boundaries prior to the $j$ th boundary, see equation (29), and whose total optical path is sufficiently close to the primary single reflection path. Under the assumption discussed above, that a maximum of three reflections is allowable, we need to include all the rays giving rise to coherent interference that reach their maximum depth inside the sample at the $i$ th boundary, for each with $i<j$ (the $i=j$ contribution is already accounted for in $\Sigma_{1}$ ) and which undergo no more than three interface reflections.

To illustrate numerically the character of equation (32) we consider the multi-layer structure depicted in figure 9 left, which contains $N^{\text {layers }}=19$ layers of thicknesses that 

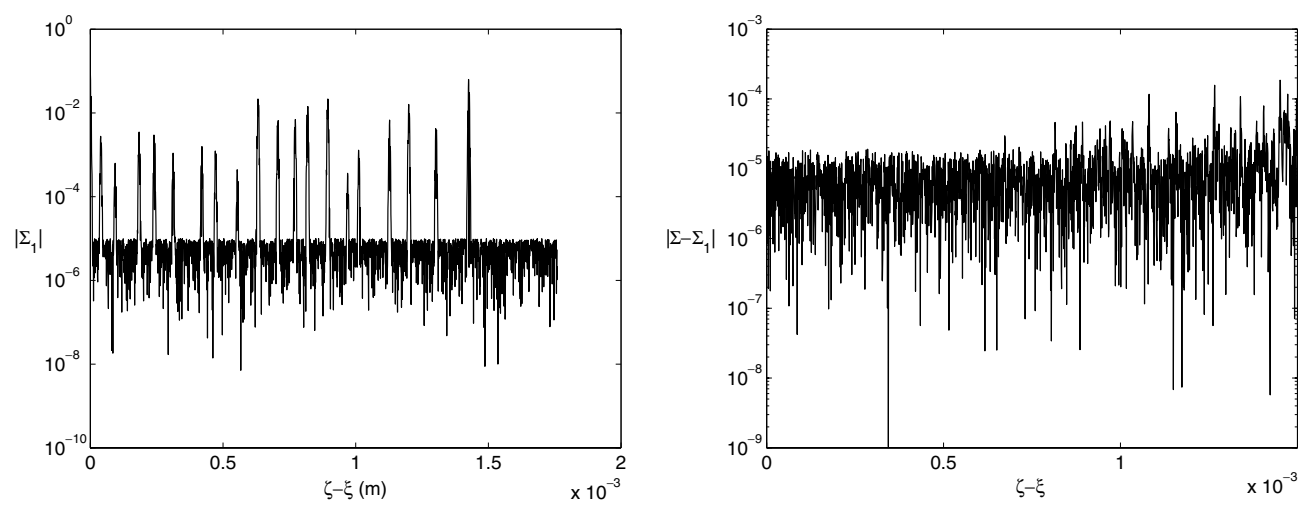

Figure 10. Left: function $\left|\Sigma_{1}(\xi)\right|$ (equation (30)), evaluated at 2200 sample positions $\xi$ for the refractive index distribution of figure 9. Right: absolute value of the difference between $\Sigma$ and its approximation $\Sigma_{1}$. Note that the error in the approximation rises above the noise floor.
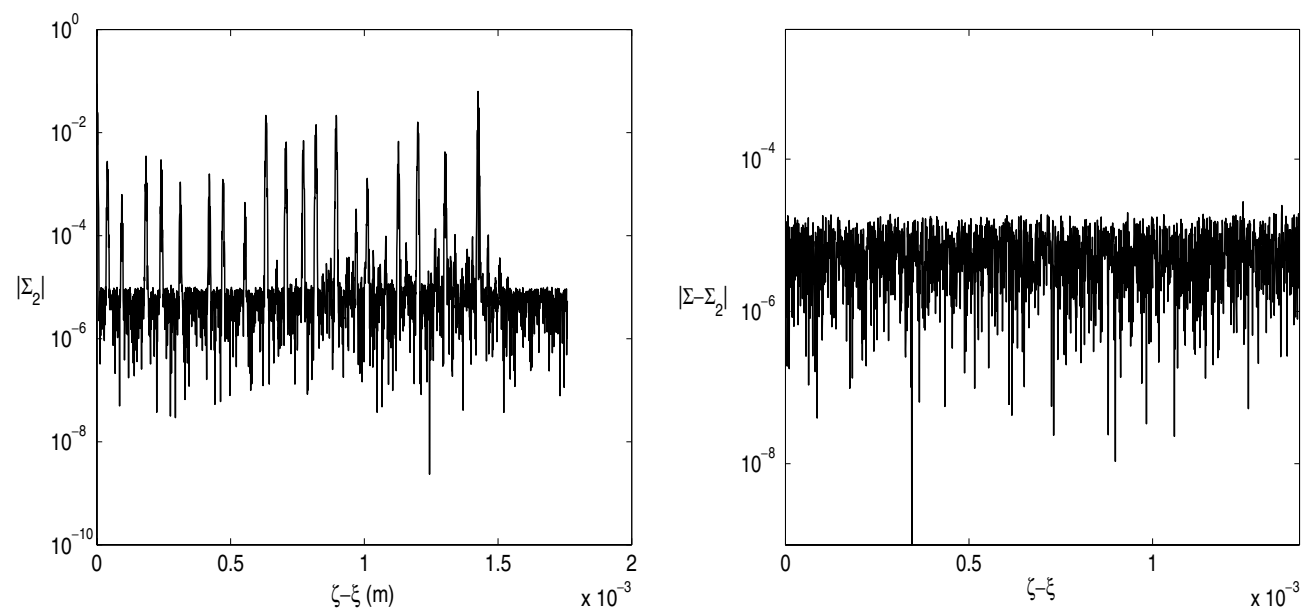

Figure 11. Left: function $\left|\Sigma_{2}(\xi)\right|$ (equation (32), with $\Sigma_{c}$ chosen to account for up to three reflections), evaluated at 2200 sample positions $\xi$ for the refractive index distribution of figure 9. Right: absolute value of the difference between $\Sigma$ and its improved approximation $\Sigma_{2}$. Note that, unlike the error in the approximation $\Sigma_{1}$, shown in figure 10, here the error is of the order of the noise floor for all sample positions.

range between $d_{1}=30 \mu \mathrm{m}$ and $d_{16}=84 \mu \mathrm{m}$; figure 9 right displays the corresponding values of $|\Sigma|$ as a function of $\xi$. Figures 10 and 11, in turn, display $\left|\Sigma_{1}\right|$ and $\left|\Sigma_{2}\right|$ as functions of $\xi$ (equations (30) and (32)), and the corresponding errors in these approximations to $|\Sigma|$. From figure 10 we see that inclusion of a single reflection does not suffice to obtain agreement of $\Sigma$ and $\left|\Sigma_{2}\right|$ up to the noise floor. Figure 11, on the other hand, shows that by adding the correction term $\Sigma_{c}$ including up to three reflections, an error below the noise floor results. Thus $\Sigma_{c}$ is an important contribution to obtain a successful model of the interference fringes.

The benefits of this approach for fast evaluation of the correlation are significant: the evaluation of $\Sigma$ by direct evaluation of integrals (19) at 2200 values of $\xi$ for the samples considered above $\left(N^{\text {layers }}=19\right)$ required a computing time of $7 \mathrm{~min}$ on a $2.4 \mathrm{GHz} \mathrm{PC}$; the accurate approximation $\left|\Sigma_{2}\right|$ at the same set of 2200 points, on the other hand, was obtained in a mere $0.14 \mathrm{~s}$. 


\subsection{Absorption averaging}

At this stage it is important to discuss the role of absorption - the imaginary part of the refractive index - in our problem. The absorption $\kappa$ has a measurable effect in the backscattered field, and, as we will show, its consideration is necessary if accurate renderings are to be obtained through the solution of OCT inverse problems. In what follows we show, however, that a precise determination of the variations of $\kappa$ within the sample is not necessary in many cases of practical interest - such as, for example, when the variations in absorption within the sample to be imaged are not too large. It is easy to see that, in such cases, only the average value of the absorption coefficient $\kappa$ plays a significant role in the backscattered returns, and thus in the determination of the real part of the refractive index throughout the sample.

To study this problem let us take a layered structure containing a number $Q$ layers of thicknesses $d_{q}$ and complex refractive indexes $N_{q}=n_{q}+\mathrm{i} \kappa_{q}(q=1, \ldots, Q)$, and let us consider a ray which travels a given physical distance $\hat{d}$ within the sample before backscattering away from it. For any such ray we have $\hat{d}=\sum_{q=1}^{Q} f_{q} d_{q}$ where $f_{q}$ denotes the number of times the ray travelled through the $q$ th layer; the corresponding scattered field, in turn, is given by

$$
E^{\text {back }}=R E_{0} \mathrm{e}^{-k_{z} \sum_{q=1}^{Q} \kappa_{q} f_{q} d_{q}} \mathrm{e}^{\mathrm{i} k_{z} z} \mathrm{e}^{\mathrm{i} k_{z} \sum_{q=1}^{Q} n_{q} f_{q} d_{q}},
$$

where the quantity $R$ involves products of the coefficients $t_{q, q+1}, r_{q, q+1}$ of equations (6) and (7).

Equation (33) can be re-expressed as follows:

$$
\begin{aligned}
E^{\mathrm{back}} & =R E_{0} \mathrm{e}^{-k_{z} \hat{d}\left(\sum_{q=1}^{Q} \kappa_{q} f_{q} d_{q}\right) / \hat{d}} \mathrm{e}^{\mathrm{i} k_{z} z} \mathrm{e}^{\mathrm{i} k_{z} \sum_{q=1}^{Q} n_{q} f_{q} d_{q}} \\
& =R E_{0} \mathrm{e}^{-k_{z} \bar{k} \hat{d}} \mathrm{e}^{\mathrm{i} k_{z} z} \mathrm{e}^{\mathrm{i} k_{z} \sum_{q=1}^{Q} n_{q} f_{q} d_{q}},
\end{aligned}
$$

where $\bar{\kappa}=\left(\sum_{q=1}^{Q} \kappa_{q} f_{q} d_{q}\right) / \hat{d}$ is the absorption average along the path visited by the ray. From (6) and (7), and since the absorptions $\kappa_{1} \ldots \kappa_{Q}$ are much smaller than the corresponding refractive index values $n_{1} \ldots n_{Q}$, we have

$$
\begin{aligned}
& t_{q, q+1} \approx \tilde{t}_{q, q+1}=\frac{2 n_{q}}{n_{q}+n_{q+1}}, \\
& r_{q, q+1} \approx \tilde{r}_{q, q+1}=\frac{n_{q}-n_{q+1}}{n_{q}+n_{q+1}},
\end{aligned}
$$

so that $R \approx \tilde{r}$ where $\tilde{r}$ is a coefficient involving products of $\tilde{t}_{q, q+1}, \tilde{r}_{q, q+1}$ and we therefore obtain our approximate expression for the reflected field

$$
E_{r} \approx \tilde{r} E_{0} \mathrm{e}^{-k_{z} \bar{k} \hat{d}} \mathrm{e}^{\mathrm{i} k_{z} z} \mathrm{e}^{\mathrm{i} k_{z} \sum_{q=1}^{Q} n_{q} f_{q} d_{q}},
$$

which depends on the absorption in terms of the average absorption only.

\section{Nonlinear solver}

\subsection{Nonlinear equations}

As mentioned above, the coherence properties of light, which certainly play a central role in previous OCT imaging strategies (such as those proposed in [14, 15]), can also be exploited to solve efficiently the fringes-to-indexes inverse problem under consideration. We have formulated the OCT inverse problem as follows: assuming

$$
\Sigma=\frac{1}{\sqrt{2 \pi}} \int_{-\infty}^{\infty} S(\omega) \tilde{u}^{\mathrm{ref} *}(\omega) \tilde{u}^{\mathrm{inc}}(\omega) \mathrm{d} \omega
$$


(see (10)) is known for various sample positions $\xi$-as described in detail in section 2.3determine the refractive index $n(z)$ within the sample.

Using the terminology introduced in points (i)-(iv) of section 2.3 , let us denote by $\Pi_{q}$ the number of values of $\Sigma$ above the threshold value $\Sigma^{\text {thresh }}$ contained in the primary spike associated with the $q$ th interface-recall that the set of interfaces is in one-to-one correspondence with the set of primary spikes, while the total number of spikes may be larger than the number of interfaces; see section 2.3. Further, we denote by $\xi_{i}^{q}\left(1 \leqslant i \leqslant \Pi_{q}\right)$ the sample positions associated with such relevant measurements for the $q$ th spike, so that $\Sigma\left(\xi_{i}^{q}\right)$ is the $i$ th measurement associated with the $q$ th interface, $1 \leqslant i \leqslant \Pi_{q}$.

The first spike, detailed in figure 6 left, can be used to determine the refractive index of the first layer, since the coherent backscattering from the subsequent interfaces is exponentially small. That is to say, this index of refraction can be obtained as the solution of a certain nonlinear vector equation, $F^{1}\left(X^{1}\right)=0 \in \mathbb{R}^{\Pi_{1}}$ where the vector function $F^{1}$ has components $F_{i}^{1}\left(X^{1}\right)=\Sigma^{\text {meas }}\left(\xi_{i}^{1}\right)-\Sigma_{2}\left(\xi_{i}^{1}, X^{1}\right)$, where $X^{1}$ denotes the refractive index $n_{1}$ to be calculated. More generally, once the first $q-1$ refractive indexes and the first $q-2$ widths have been found, the $q$ th spike can be used to determine the refractive index of the $q$ th layer and the thickness of the $(q-1)$ th layer.

In each case, we must determine the values of a refractive index and a thickness as the solution to a certain nonlinear equation $F^{q}\left(X^{q}\right)=0 \in \mathbb{R}^{\Pi_{q}}$ where $X^{q}$ is the two-dimensional vector

$$
X^{q}=\left(n_{q}, d_{q-1}\right)
$$

Here as above

$$
F_{i}^{q}\left(X^{q}\right)=\Sigma^{\text {meas }}\left(\xi_{i}^{q}\right)-\Sigma\left(\xi_{i}^{q}, X^{q}\right) .
$$

While in principle it would suffice to use two equations to determine $n_{q}$ and $d_{q-1}$, we have found that, as is common in the solution of inverse problems, use of an overdetermined system of $\Pi_{q}$ equations and two unknowns is highly advantageous in our problem as well. Denoting by $X_{0}^{q}$ the least-squares solution of the overdetermined system of nonlinear equations $F\left(X^{q}\right)=0$ or, equivalently, the minimizer of the functional $\phi^{q}\left(X^{q}\right)=\frac{1}{2} F^{q}\left(X^{q}\right)^{T} F^{q}\left(X^{q}\right)$, we obtain $X_{0}^{q}$ by means of the Gauss-Newton method: defining by $Y_{n}^{q}$ is the solution of the linear system

$$
A_{n}^{q} Y_{n}^{q}=-\nabla \phi^{q}\left(X_{n}^{q}\right)
$$

(where $\nabla \phi^{q}\left(X_{n}^{q}\right)=J_{F^{q}}^{T}\left(X_{n}^{q}\right) F^{q}\left(X_{n}^{q}\right), A_{n}^{q}=J_{F^{q}}^{T}\left(X_{n}^{q}\right) J_{F^{q}}\left(X_{n}^{q}\right), J$ the Jacobian of $F^{q}$ ), we produce the $(n+1)$ th Gauss-Newton iterate by means of the expression

$$
X_{n+1}^{q}=X_{n}^{q}+Y_{n}^{q} .
$$

As it happens, the derivatives necessary to evaluate the gradient and the Jacobian of the function $\phi^{q}$ corresponding to the $q$ th interface can be computed analytically from expression (32) for the multiple-scattering approximations $\Sigma_{2}$ of the correlation $\Sigma$. Indeed, note that, when working on the $q$ th interface, the only term in equation (32) that depends on the differentiation variables $n_{q}$ and $d_{q-1}$ is $\Sigma_{1}$, as given in equation (30). The term $\Sigma_{c}$ only involves reflections arising from the previous interfaces and therefore it does not depend on either $n_{q}$ or $d_{q-1}$.

A difficulty arises in our minimization problem since the Gauss-Newton method generally converges to a local minimum only, and local minima are ubiquitous in our problem. An important task is, then, to find appropriate starting points which guarantee that a Newtonbased method yields the global minimum. Our construction of an appropriate class of starting points, which is presented in the following section, is indeed what renders our approach feasible. 


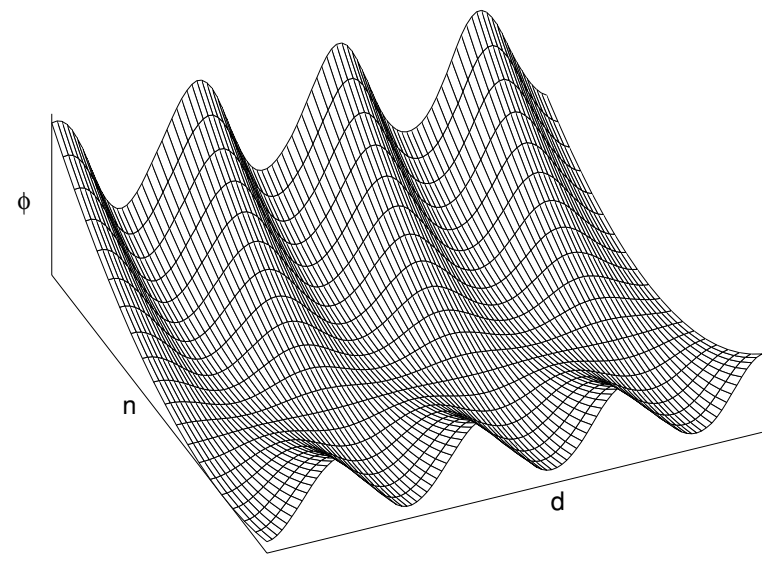

Figure 12. Mean-square function $\phi^{q}=\phi^{q}(n, d)$.

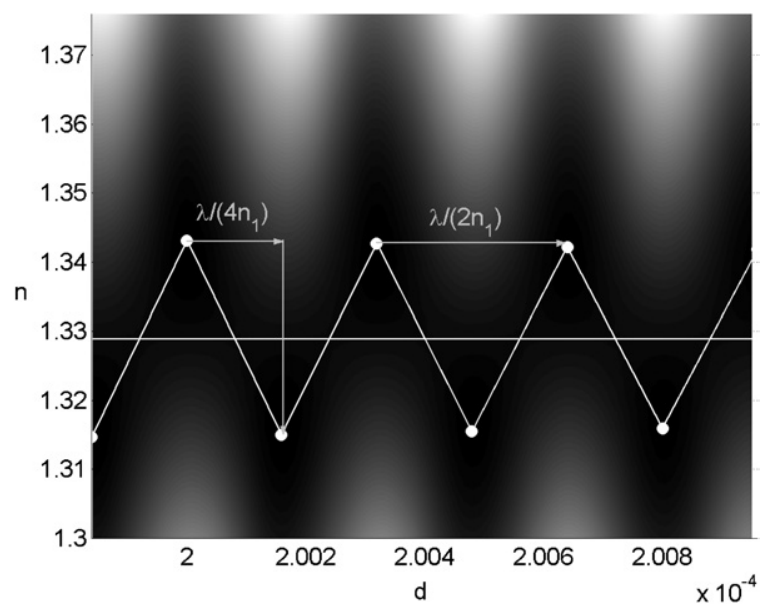

Figure 13. Mean-square function $\phi^{q}=\phi^{q}(n, d)$ : dark and light areas indicate small and large function values, respectively.

\subsection{Structure of the minimization problem and selection of initial guesses}

Our algorithm for determination of global minima is based on some important general properties of the functions $\phi^{q}\left(X^{q}\right)$ which are illustrated in figures 12 through 15:

(i) The function $\phi^{q}(X)$ has several local minima within the minimization domain, see figure 12 and the top-view figure 13, in which darker shades identify smaller values of the function $\phi$.

(ii) For fixed values of $d$ the function $\phi^{q}$ exhibits a parabolic behaviour with respect to refractive index variations (see figure 14) and, for fixed values of $n$, it exhibits oscillatory behaviour with respect to variations of the thickness parameter $d$, (see figure 15).

(iii) The local minima (resp local maxima) of $\phi^{q}$ as a function of $d$ for fixed $n>n_{q-1}$ are located at positions that vary only slightly with $n$, and the distance between two consecutive local minima (resp local maxima) is $\approx \tilde{\lambda} / 2$, where $\tilde{\lambda}=\lambda / n_{q-1}$ is the centre wavelength in the $(q-1)$ th layer $(\bar{\omega}=2 \pi c / \lambda$ is the centre frequency of the laser). 


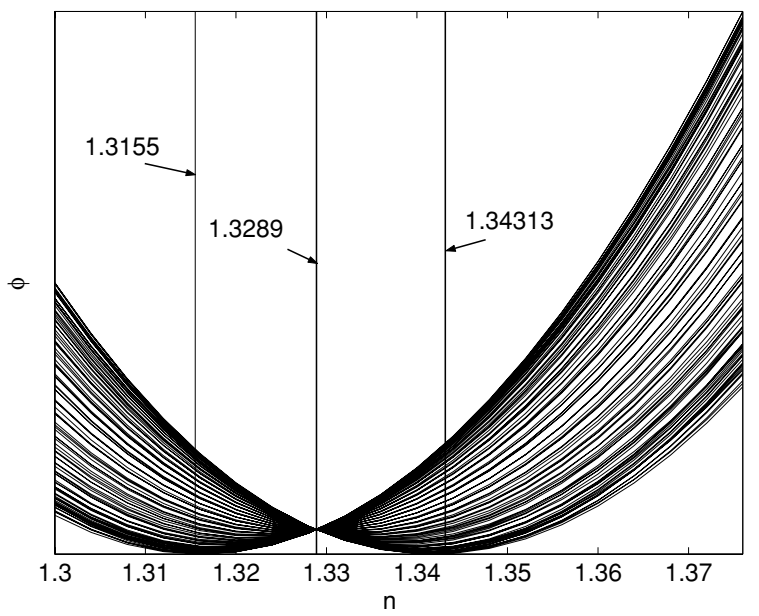

Figure 14. Mean-square function $\phi^{q}$ for several values of the refractive index $n$.

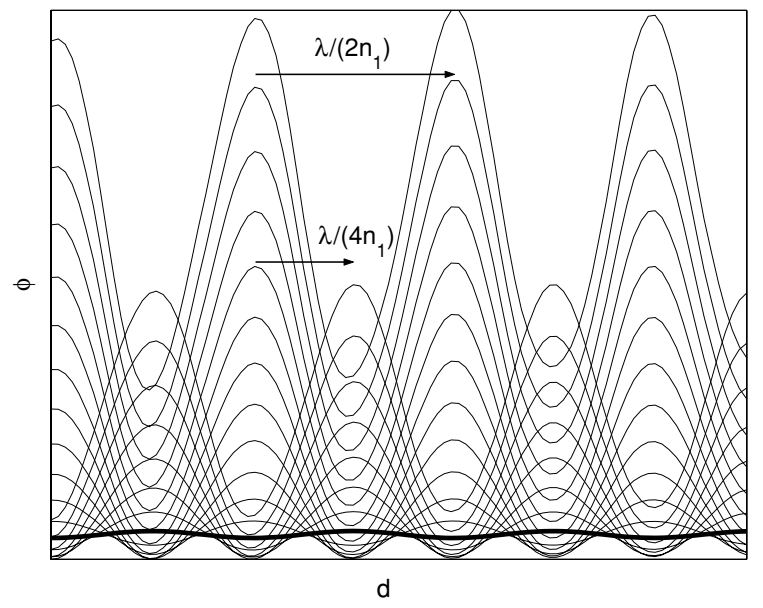

Figure 15. Mean-square function $\phi^{q}(n, d)$ as a function of $d$ for several values of $n$.

Analogously, for all fixed $n<n_{q-1}$ the distance between two consecutive local minima or maxima is, again, $\approx \tilde{\lambda} / 2$. The fact that the distance between local maxima/minima is approximately half a wavelength can be understood easily by considering that, as the position of the interface under consideration is perturbed by an amount $\pm e$, the phase of the returning reflection beam undergoes a perturbation of $\pm 2 e$, i.e, a ray travelling through the sample and returning from the interface needs to travel an additional $\pm 2 e$ to arrive at the detector.

(iv) The symmetries observed in figure 13 can be understood by considering a two-layer problem, with refractive indexes $n_{1}$ and $n_{2}$ on the left and right of the interface, respectively, and illuminated by a normally incident ray, as depicted in figure 16 . The field reflected at the interface between the two layers is determined by the reflection coefficient $r=\left(n_{1}-n_{2}\right) /\left(n_{1}+n_{2}\right)$, and thus, for a fixed value of $n_{1}$ and setting $n_{2}=n_{1}+e$, to first order in $e$ the field reflected by the interface is an odd function of $e$,

$$
\frac{-e}{2 n_{1}-e}=\frac{-e}{2 n_{1}}\left(1+e / 2 n_{1}+\cdots\right) \approx \frac{-e}{2 n_{1}} .
$$




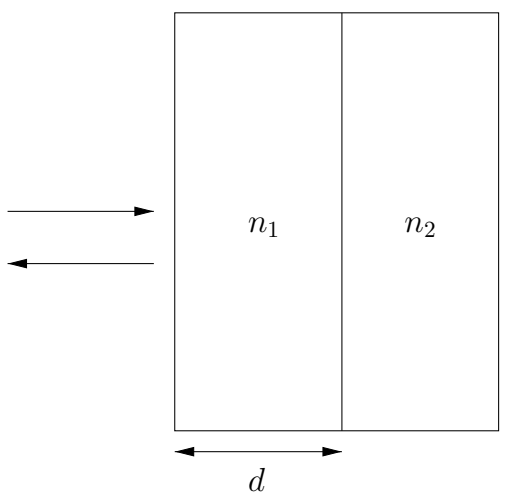

Figure 16. Two-layer structure with refractive indexes $n_{1}$ and $n_{2}$ and thickness $d$.

On the other hand, an increase of $d$ in the amount $\Delta d=\frac{\lambda}{4 n_{1}}$ leads to an increase in the phase of the corresponding portion of field backscattered from the sample by $\pi$ - that is, it leads to a change in sign in the field itself. It follows that, to first order in $e$, the quantity $\phi^{q}=\phi^{q}(n, d)$ as a function of $d$ will be invariant under the substitutions

$$
e=n-n_{1}, \quad n_{1}+e \rightarrow n, \quad d+\frac{\lambda}{4 n_{1}} \rightarrow d,
$$

which explains the symmetries in figure 13 around the line $n=n_{1}$.

(v) In view of points (ii) to (iv) above we see that the line $n=n_{q-1}$ separates two sequences of local minima, and these two sequences contain all the local minima relevant to the determination of the thickness $d_{q-1}$ and the refractive index $n_{q}$ associated with the interference spike under consideration.

In light of these properties we design our minimization algorithm as follows: to determine the thickness and refractive index associated with a given intensity spike, we begin our optimization by setting the thickness parameter $\xi$ to equal the thickness associated with the largest measured value of the correlation $\Sigma$ in that spike. Next, we perform a minimization along the refractive index direction, thus obtaining a first high-quality initial guess $p_{0}$ for the minimization problem. Then, using the initial guess $p_{0}$ we proceed with Gauss-Newton iterations to convergence, to obtain a first local minimum, which we denote by $p_{1}$. Once $p_{1}$ is obtained, we can produce very good new initial guesses in each one of the basins of attraction, see figures 12, 13 and 15, in view of properties (iv) and (v) above, by simply using the symmetry with respect to $n=n_{1}$ and the fact that the $n$-axis distances between minima are known with relatively small uncertainties. The Newton iterations starting from each one of these initial guesses converge rapidly to local minima $p_{1}, p_{2}, \ldots, p_{m}$. The global minimization problem is then solved by selecting the $p_{i}$ with a minimum $\phi$ value.

\section{Numerical results}

In this section we demonstrate the performance of our algorithms through a variety of numerical examples. In all cases an error was added to synthetic 'experimental data' so as to simulate the experimental noise floor; the added noise floor was taken to be random and to the order of $10^{-5}$, relative to a unit incident field $\left(10^{-4}\right.$ relative to the largest interference intensity), see section 2.3. This noise level is much larger than the various noise bars reported in the literature $[15,23-26]$. In our numerical examples we use the reasonable but otherwise arbitrary values 

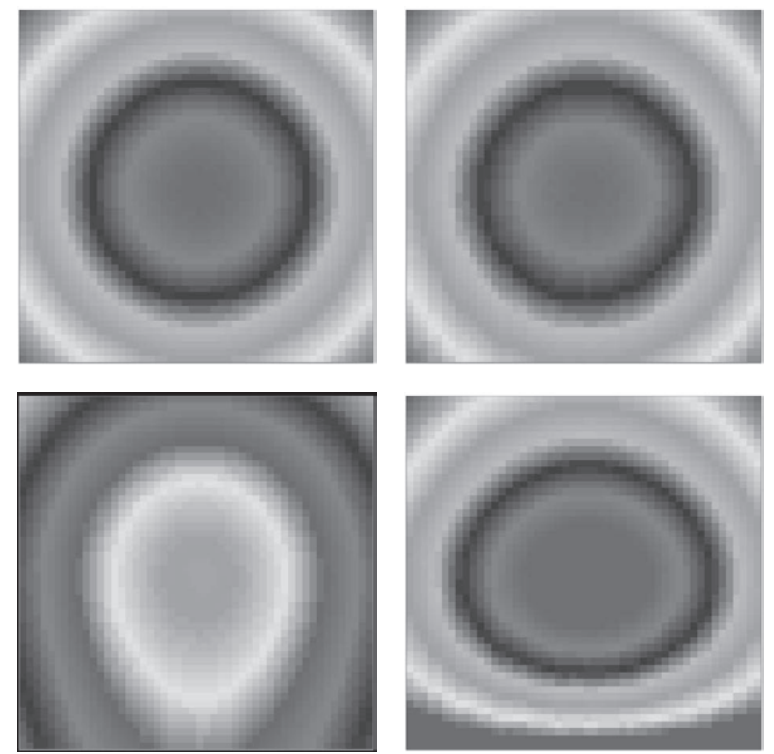

Figure 17. Effect of absorption averaging. Upper left: prescribed refractive index map. Upper right, lower left and lower right: reconstructions using values $\kappa=0.6 \times 10^{-4}, \kappa=0$ and $\kappa=1.2 \times 10^{-4}$ for the average absorption parameter, respectively.

$\lambda=850 \mathrm{~nm}, \Delta \lambda=70 \mathrm{~nm}$ [22], together with the corresponding values of $\bar{w}$ and $\Delta \omega$. The parameter $\varepsilon$ in the scanning process was taken to equal $\varepsilon=800 \mathrm{~nm}$ : close to but different from the $850 \mathrm{~nm}$ centre wavelength of the source; see section 2.3 for a discussion on useful choices of the parameter $\varepsilon$. Throughout this section, unless otherwise specified, all lengths (e.g. the $d$ axes) are measured in metres (m).

\subsection{Absorption effects}

To appreciate the effect of the approximations introduced in section 2.5 , we present solutions of an inverse problem containing a wide range of absorption variations, with an average absorption value equal to $0.6 \times 10^{-4}$. We generally may not assume this average value is known, and thus reconstructions should proceed through appropriate tuning of this parameter, see figure 17.

This figure presents a prescribed refractive index map $(50 \times 50$ square pixels $)$ together with an image reconstructed under various assumptions for the average absorption parameter. Each one of the reconstructed figures resulted from solution of 50 columnar inverse problems, each one of which requires determination of 50 refractive index values. We see that, as anticipated, values of the absorption parameter close to the actual average absorption value result in excellent reconstructions, and that even reconstructions based on values of this parameter far from the actual average absorption value produce informative images, albeit somewhat distorted.

In our second set of numerical examples we present inversion results corresponding to the 19-layer structure shown in figure 18 top left, under the assumption of three different (random) arrays of absorption coefficients, shown in figure 18 top right, bottom left and bottom right. In all three cases the absorption coefficients $\kappa$ range between $10^{-5}$ and $10^{-4}$. The three corresponding reconstructions, obtained under assumption of a constant absorption 

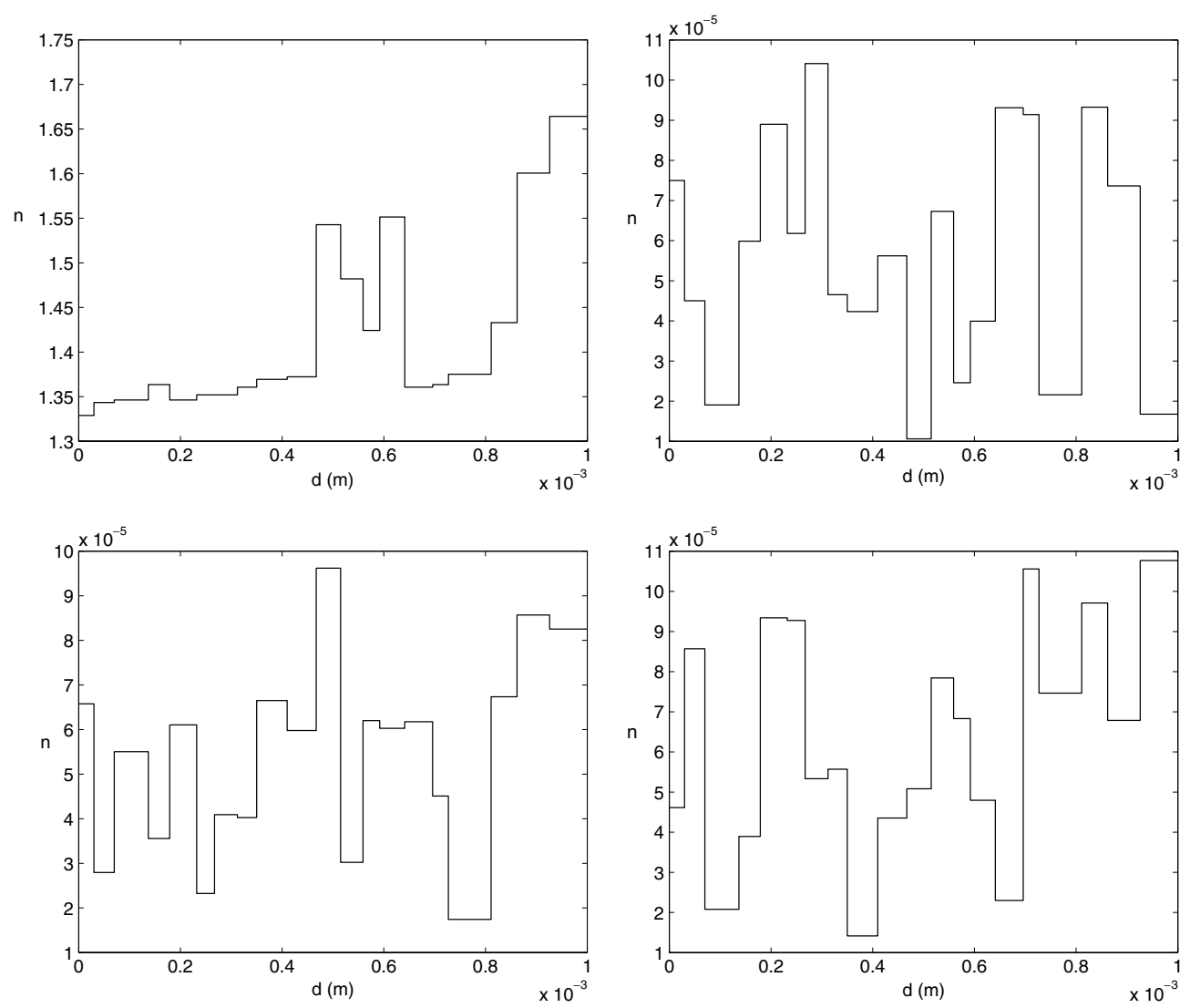

Figure 18. 19-layer structure. Upper left: refractive index distribution. Top right, bottom left and bottom right: three different (random) absorption distributions.

equal to the average absorption in the true samples (which equals $0.6 \times 10^{-4}$ ) are shown in figure 19. Each one of these curves was obtained in a run of approximately $0.4 \mathrm{~s}$ on a $2.4 \mathrm{GHz}$ Pentium IV processor.

\subsection{Large absorption/noise values}

Next we present examples that demonstrate the behaviour of our solver in the presence of large values of absorption and noise. In the first pair of examples we assume samples whose refractive indexes are given by the top left portions of figure 18, and whose absorptions are ten times those depicted in figure 18 top right. Figure 20 left, displays the corresponding values of the correlation $|\Sigma|$. We see that a significant loss of information occurs, as the increased absorption gives rise to measured values of the correlation which fall below the noise level. Figure 20 left displays the corresponding solution produced by our solver in this case. Clearly, only portions of the sample for which the measurements are not polluted by noise can be reconstructed with any accuracy.

In the second pair of examples relating to absorption we reduce the absorption level back to the values of figure 18, and study the effect of an increased noise floor by assuming the random noise is of the order of $10^{-4}$ - a factor of 10 higher than all other values assumed 


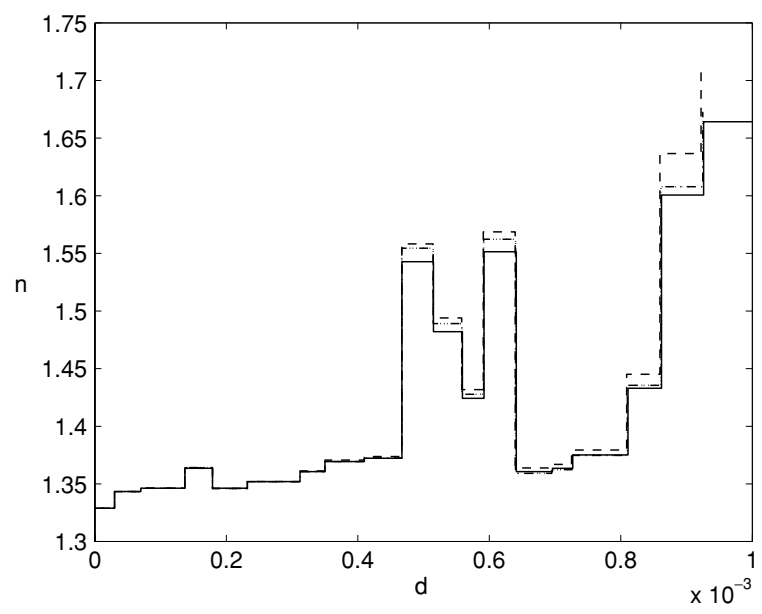

Figure 19. 19-layer structure. Refractive index distribution (solid) and the corresponding reconstruction for three different absorption values distributions.
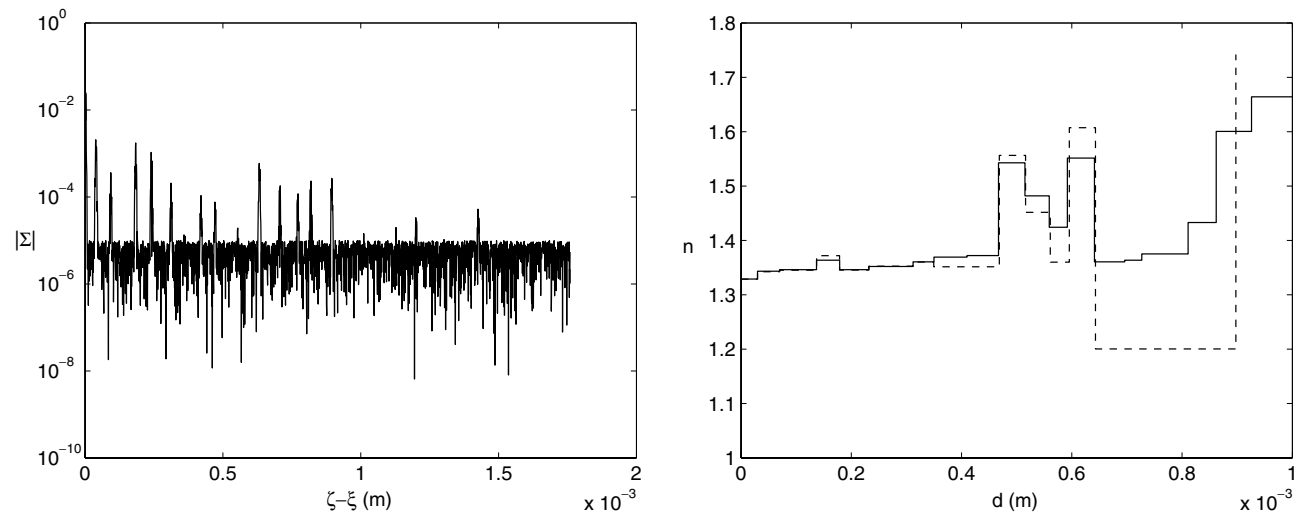

Figure 20. Layered medium with absorption values $\kappa$ ranging between $10^{-4}$ and $10^{-3}$. Left: $|\Sigma|$. Right-continuous: actual refractive index $n$. Right-dashed: reconstruction of $n$.

in this text, and usually found in practice. Figure 21 left shows the corresponding measured values of $|\Sigma|$ for the 19-layer structure. We note that the noise floor in these cases equals the threshold value $\Sigma^{\text {thresh }}=10^{-4}$ used for the detection of interfaces. Still, reasonable solutions are obtained - at least for the more highly illuminated portions of the sample.

\subsection{Volumetric imaging}

As mentioned in section 1, the inverse solver developed in this work does not apply directly to the problem of imaging of volumetric samples containing non-planar interfaces. To gain an insight on the type of performance that might be expected when extending these methods to such three-dimensional configurations, in this section we present the results of applications of our algorithm to an imaging problem involving a true cell geometry, in which the volumetric geometry has been replaced by a 'chessboard approximation', that is, an array of square pixels which are then treated, as in section 4.1 , as sequences of columnar one-dimensional inverse 

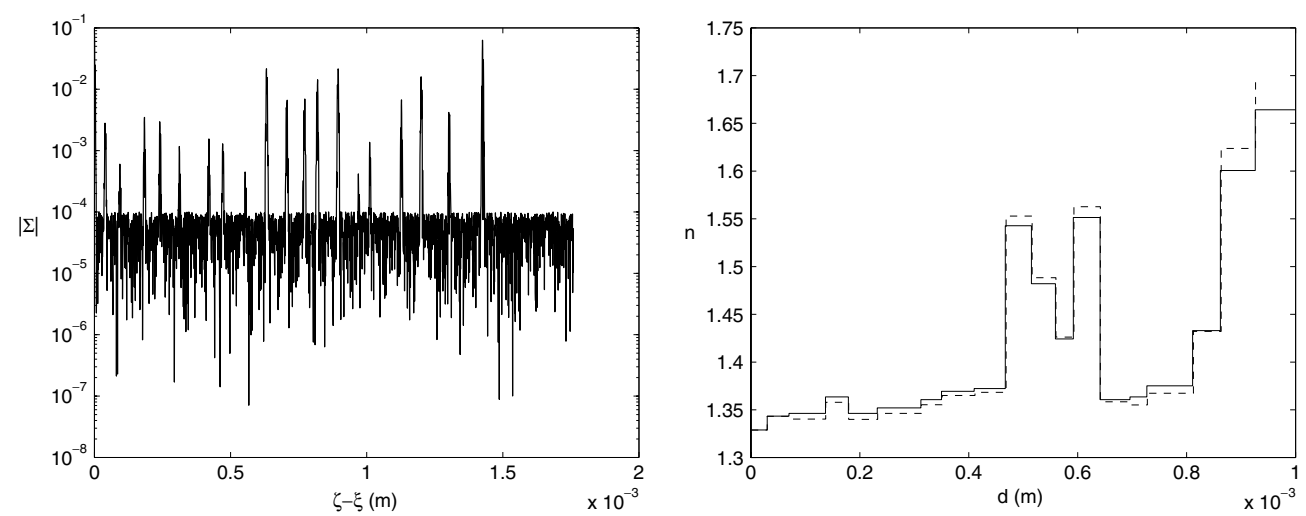

Figure 21. Reconstruction of a 19-layer structure under a noise level of the order of $10^{-3}$ relative to the maximum interference fringe intensity (which is itself of the order of $10^{-1}$ ). Left: $|\Sigma|$. Right: corresponding solution for this noise level.
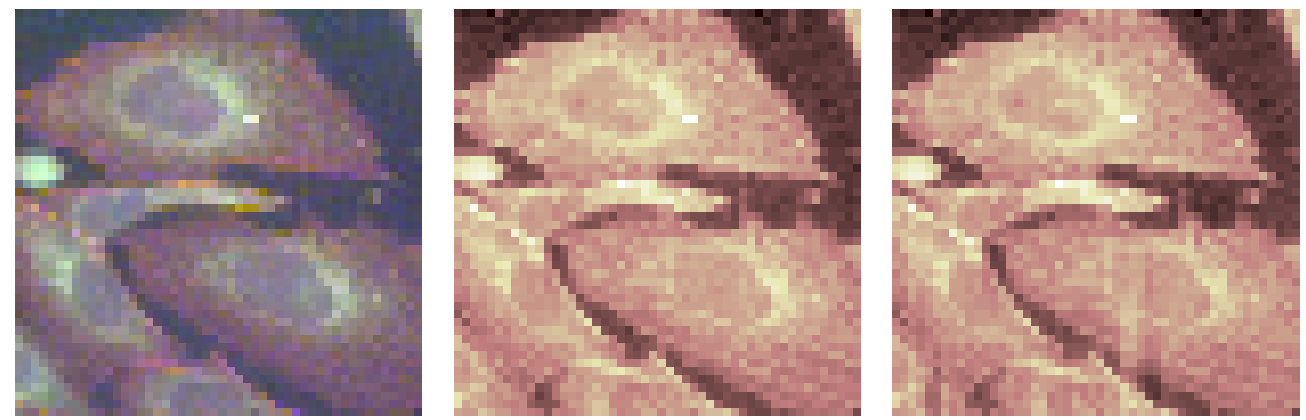

Figure 22. Left: chessboard rendering of an original cell figure. Centre: refractive index assignment. Right: reconstruction.

(This figure is in colour only in the electronic version)

problems. In detail, in figure 22 the $x$-axis is horizontal and the $z$-axis is vertical, and each one of the 50 vertical columns (each one of which, in turn, contains 50 pixels), is considered as defining a single OCT inverse problem for a layered structure containing 50 layers (51 interfaces). Each one of the pixels is square in shape, and the overall structure is assumed to measure $10^{-3} \mathrm{~m} \times 10^{-3} \mathrm{~m}$ in size.

This geometry was obtained from http://dept.kent.edu/projects/cell/images.html; the needed 'chessboard' approximations of this geometry are displayed in the left portion of figure 22. The $50 \times 50$ chessboard structures shown here reflect the level of detail that can be produced under the value of the coherence length assumed in this work, $l_{c}=10.3 \mu \mathrm{m}$ (see equation (21)). Finer resolutions can certainly be obtained, provided light sources with correspondingly reduced coherence lengths are used.

The colour code in the left portion of figure 22 matches that of the figure in the website mentioned above. The centre portion of this figure, in turn, displays our own colour code, which represents a value of the refractive index between $n=1.3$ and $n=1.8$ and absorptions of the order of $10^{-5}<\kappa<10^{-4}$-all fairly common values for biological applications. (The refractive index value at a given pixel in the centre figure was chosen as a linearly scaled version of the red component in the RGB true-colour image map for the corresponding left 
figures.) The right figure displays the results of our reconstruction. Note again that the righthand figure resulted from solution of 50 inverse problems, one for each of the 50 columns making up our sample; this procedure produced the 2500 refractive index values depicted in the right portion of figure 22 . The reconstruction is nearly perfect.

\section{References}

[1] Bouma B E and Tearney G J (ed) 2002 Handbook of Optical Coherence Tomography (New York: Dekker)

[2] Tearney G J, Brezinski M E, Southern J F, Bouma B E, Hee M R and Fujimoto J G 1995 Determination of the refractive index of highly scattering human tissue by optical coherence tomography Opt. Lett. $202258-60$

[3] Hettinger J W, de la Peña Mattozzi M, Myers W R, Williams M E, Reeves A, Parsons R L, Haskell R C, Petersen D C, Wang R and Medford J I 2000 Optical coherence microscopy. A technology for rapid in vivo non-destructive visualization of plants and plant cells Plant Physiol. 123 3-15

[4] Bruno O P and Chaubell J 2003 Inverse scattering problem for optical coherence tomography Opt. Lett. 28 2049-51

[5] Fukano T and Yamaguchi I 1999 Separation of measurement of the refractive index and the geometrical thickness by use of a wavelength-scanning interferometer with a confocal microscope Appl. Opt. 38 4065-73

[6] Fukano T and Yamaguchi I 1996 Simultaneous measurement of thicknesses and refractive indices of multiple layers by a low-coherence confocal interference microscope Opt. Lett. 21 1942-4

[7] Watanabe Y and Yamaguchi I 2002 Geometrical tomography imaging of refractive indices through turbid media by a wavelength-scanning heterodyne interference confocal microscope Appl. Opt. 41

[8] Gelfand I M and Levitan B M 1951 On the determination of a differential equation from its spectral function Am. Math. Soc. Transl. 2 253-304

[9] Hagin F 1981 Some numerical approaches to solving one-dimensional inverse problems J. Comput. Phys. 43 16-30

[10] Chen Y and Rokhlin V 1992 On the inverse scattering problem for the Helmholtz equation in one dimension Inverse Problems 8 365-91

[11] Jackson J D 1999 Classical Electrodynamics (New York: Willey)

[12] 1972 American Institute of Physics American Institute of Physics Handbook B H Billing et al (section editors), D E Gray (coordinate editors) (New York: McGraw Hill)

[13] Kaye G W C 1995 Tables of Physical and Chemical Constants (Harlow: Longman)

[14] Huang D, Swanson E, Lin C P, Schuman J S, Stinson W G, Chang W, Hee M R, Flotte T, Gregory K, Puliafito C A and Fujimoto J G 1991 Optical coherence tomography Science 254 1178-81

[15] Youngquist R, Carr S and Davies D 1987 Optical coherence-domain reflectometry: a new optical evaluation technique Appl. Opt. 26 158-60

[16] Hoeling B M, Fernandez A D, Haskell R C, Huang E, Myers W R, Petersen D C, Ungersma S E, Wang R, Williams M E and Fraser S E 2000 An optical coherence microscope for 3 dimensional imaging in developmental biology Opt. Exp. 6 136-46

[17] Chew W C 1990 Waves and Fields in Inhomogeneous Media (Princeton, NJ: Van Nostrand Reinhold)

[18] Hecht E and Zajac A 1979 Optics (Princeton, NJ: Addison-Wesley)

[19] http://etd.caltech.edu/etd/available/etd-09092003-212358/thesis

[20] Goodman J W 1985 Statistical Optics (New York: Wiley)

[21] Born M and Wolf E 1964 Principle of Optics (London: MacMillan)

[22] Fercher A F, Drexler W, Hitzenberger C K and Lasser T 2003 Optical coherence tomography: principles and applications Rep. Prog. Phys. 66 239-303

[23] Gilgen H H, Novăk R P, Salathé R P, Hodel W and Béaud P 1989 Submillimeter optical reflectometry J. Ligthwave Tech. 7 1225-33

[24] Yadlowsky M, Schmitt J and Bonner R 1995 Multiple-scattering in optical coherence microscopy Appl. Opt. 34 5699-707

[25] Takada K, Yokohama I, Chida K and Noda J 1987 New measurement system for fault location in optica wave-guide based on devices on an interferometric technique Appl. Opt. 26 1603-6

[26] Izatt J, Hee M, Owen G, Swason E and Fujimoto J 1994 Optical coherence microscopy in scattering media Opt. Lett. 19 590-2 